\title{
Testing for Unit Roots with Flow Data and Varying Sampling Frequency
}

\author{
Marcus J. Chambers \\ University of Essex
}

March 2001

\begin{abstract}
This paper considers tests for a unit root in a flow variable when the span of data and/or the sampling frequency are allowed to vary. The limiting distributions of the statistics are obtained under both the null and alternative hypotheses, thereby enabling an analysis of the consistency properties of the tests to be conducted. Contrary to the situation with a stock variable, it is found that it is possible to consistently test for a unit root in a flow variable even when the span of the data is fixed, and, furthermore, that increasing the span of the data is not in itself sufficient for consistent testing. Some new simulation results are provided while the theoretical results obtained help to explain recent simulation findings by other authors involving unit root tests with flow variables.
\end{abstract}

Key words: flow variable; sampling frequency; unit root test.

Acknowledgements: I would like to thank Roy Bailey and Rex Bergstrom for helpful comments on an earlier version of this piece of work. Financial support from the Economic and Social Research Council under grant number R000222961 is gratefully acknowledged.

Address for Correspondence: Professor Marcus J. Chambers, Department of Economics, University of Essex, Wivenhoe Park, Colchester, Essex CO4 3SQ, England. Tel: +44 1206 872756. Fax: +44 1206 872724. E-mail: mchamb@essex.ac.uk.

URL: http://privatewww.essex.ac.uk/ ${ }^{2}$ mchamb 


\section{Introduction}

Although there is an enormous literature on testing for a unit root in a time series, only a relatively small amount of this research has been concerned with the differing ways in which the variable itself can be observed. By far the most intensively researched topic in this category is testing for unit roots in processes observed at seasonal frequencies, giving rise to the possibility of seasonal unit roots in addition to the more common zero frequency unit root. But there are also more fundamental differences in the ways in which variables are recorded that arise due to their very nature. Some variables, such as prices, interest rates, and the capital stock, can in principle be observed at any given point in time, while others, such as consumption expenditures, income and dividends, are observed as the accumulation of an underlying rate of flow over a given time interval. While the effects of such temporal aggregation are well known in the estimation of (at least asymptotically) stationary systems, such effects have received rather less attention in the unit root literature. This paper aims to fill at least part of that gap.

The issue of unit root test consistency when the sampling frequency is allowed to vary was analysed by Perron (1991). He dealt with a stock variable generated by an OrnsteinUhlenbeck process and considered the effects of allowing data span and/or sampling frequency to vary. One of his main findings was that a test for a unit root is only consistent if the span of data is allowed to increase. This implies that it is not sufficient merely to rely on more frequent observations for a given span (and hence a larger sample) in order for a test to be consistent. So far, however, there appear to be no theoretical results for the case in which the variable is a flow and is observed as the accumulation of the underlying rate over the sampling interval, although two simulation studies have been published on this topic, by Choi (1992) and by $\mathrm{Ng}$ (1995). These simulation studies are discussed in more detail later in this paper in the light of the theoretical results obtained. One might be tempted to conjecture that the way in which a variable is sampled (as a stock or a flow) will have no bearing on the properties of unit root tests. But, as will be seen, this is not so. The temporal aggregation inherent in observing a flow variable acts not only to induce a moving average disturbance but also to change the order of magnitude of the variance (in terms of sampling frequency) as compared to a stock. If $h$ denotes the sampling interval, e.g. a year or a quarter, then the variance of a flow is $O\left(h^{3}\right)$ as $h \downarrow 0$, compared with $O(h)$ for a stock. As a consequence, the orders of magnitude of various sample moments involving flow data are different to the stock case, and the predictions of Perron (1991) in the case of a stock variable no longer hold. In particular, it is possible to obtain a consistent test of a unit root 
with a flow variable even when the span of data is fixed, and, furthermore, increasing the span is not in itself sufficient for a consistent test to exist.

The paper is organised as follows. Section 2 defines the model and derives the behaviour of the observed flow variable under both the null and alternative hypotheses. Sections 3 and 4 derive the limiting distributions of the ordinary least squares (OLS) and instrumental variables (IV) estimators under the null and alternative hypotheses, respectively, while section 5 analyses the consistency properties of the unit root tests. Section 6 reports some simulation results and also discusses the simulation results of Choi (1992) and $\mathrm{Ng}$ (1995) in the context of the theoretical results obtained here. Section 7 concludes, while the Appendix contains the proofs of all lemmas, theorems and propositions contained in the paper.

\section{The model}

The variable of interest, $y$, is assumed to satisfy the Ornstein-Uhlenbeck process

$$
d y(t)=\gamma y(t) d t+\sigma d w(t), \quad t>0
$$

where $w(t)$ denotes a standard Wiener process, $0<\sigma<\infty$, and the initial value $y(0)$ is assumed to be fixed. Observations on $y(t)$ are made at intervals of length $h>0$ over the interval $0<t \leq N$, where $N$ denotes the span of the data. The number of observations is therefore given by $T=N / h$. Note that $h$ denotes the sampling interval while $1 / h$ denotes the sampling frequency. Thus if the unit of time denotes a year and the data are observed quarterly, then the sampling interval is $h=1 / 4$ and the sampling frequency is $1 / h=4$ (observations per year). When $y$ is a flow variable the observations take the form of integrals of $y(t)$ over each observation interval of length $h$, denoted

$$
y_{t h}=\int_{t h-h}^{t h} y(r) d r, \quad t=1, \ldots, T \text {. }
$$

The observed sequence is therefore $y_{h}, y_{2 h}, \ldots, y_{T h}$.

The null hypothesis of interest corresponds to $\gamma=0$ in (1), in which case $y(t)$ evolves as a random walk in continuous time. The two-sided alternative is that $\gamma \neq 0$. When $\gamma>0, y(t)$ is explosive, while if $\gamma<0, y(t)$ is stable. Tests of the null hypothesis are conducted using the observed discrete time data, whose law of motion, under both the null and alternative hypotheses, is given in Theorem 1. 
Theorem 1. (a) Under the alternative hypothesis $\gamma \neq 0$, the discrete time flow data generated by (1) satisfy the difference equation

$$
y_{t h}=\alpha_{h} y_{t h-h}+u_{t h}, \quad t=1, \ldots, T
$$

where $\alpha_{h}=e^{\gamma h}$ and $u_{t h}$ is defined by

$$
u_{t h}=\frac{\sigma}{\gamma}\left[\int_{0}^{h}\left(e^{\gamma s}-1\right) d w(t h-s)+\int_{0}^{h}\left(e^{\gamma h}-e^{\gamma s}\right) d w(t h-h-s)\right] .
$$

The autocovariance properties of $u_{t h}$ are given by

$$
\begin{aligned}
& E\left(u_{t h}^{2}\right)=\gamma_{0}(h)=\frac{\sigma^{2}}{\gamma^{2}}\left[h\left(e^{2 \gamma h}+1\right)+\frac{1-e^{2 \gamma h}}{\gamma}\right]=\frac{2}{3} \sigma^{2} h^{3}+O\left(h^{4}\right), \\
& E\left(u_{t h} u_{t h-h}\right)=\gamma_{1}(h)=\frac{\sigma^{2}}{\gamma^{2}}\left[\frac{e^{2 \gamma h}-1}{2 \gamma}-h e^{\gamma h}\right]=\frac{1}{6} \sigma^{2} h^{3}+O\left(h^{4}\right),
\end{aligned}
$$

while $E\left(u_{t h} u_{t h-k h}\right)=0$ for $k \geq 2$.

(b) Under the null hypothesis $\gamma=0$, the discrete time flow data generated by (1) satisfy the difference equation

$$
y_{t h}=y_{t h-h}+u_{t h}, \quad t=1, \ldots, T,
$$

where $u_{t h}$ is defined by

$$
u_{t h}=\sigma\left[\int_{0}^{h} s d w(t h-s)+\int_{0}^{h}(h-s) d w(t h-h-s)\right] .
$$

The autocovariance properties of $u_{t h}$ are given by $E\left(u_{t h}^{2}\right)=\frac{2}{3} \sigma^{2} h^{3}, E\left(u_{t h} u_{t h-h}\right)=\frac{1}{6} \sigma^{2} h^{3}$, while $E\left(u_{t h} u_{t h-k h}\right)=0$ for $k \geq 2$.

The discrete time flow data therefore satisfy an $\operatorname{ARMA}(1,1)$ process when $\gamma \neq 0$ while the first differences are MA(1) when $\gamma=0$. Note that the autoregressive parameter $\alpha_{h}>1$ when $\gamma>0$ and $0<\alpha_{h}<1$ when $\gamma<0$. Note, too, that when $h \downarrow 0$ it follows that $\alpha_{h} \rightarrow 1$ regardless of whether $\gamma$ is positive or negative. Theorem 1 immediately highlights a crucial difference between the models satisfied by flow data and stock data. With stock data, ${ }^{1}$ observations are of the form $y_{t h}=y(t h)$, rather than in the form of an integral, and although the autoregressive coefficient is $e^{\gamma h}$ in both cases, the disturbance is white noise in

\footnotetext{
${ }^{1}$ For results in the case of a stock variable, see Perron (1991) and section 6 of Phillips (1987a).
} 
the case of a stock, whereas it is $\mathrm{MA}(1)$ for a flow. But there is also a more fundamental difference. Theorem 1 shows that the variance and autocovariance of the disturbance are $O\left(h^{3}\right)$ for a flow, but Perron (1991) has shown that for a stock the variance is $O(h)$. This difference in the order of magnitude of the variance of the process arises because of the integrating filter that is applied to flow variables to yield the observations. Whether this has an effect on estimation and testing is investigated below.

The asymptotic analysis that follows is concerned with the behaviour of certain estimators and test statistics as $h \downarrow 0$ and/or $N \uparrow \infty$. It is convenient to index $h, N$ (and T) by $n$, as in Phillips (1987a) and Perron (1991), so that $T_{n}=N_{n} / h_{n}$, and to allow $n \uparrow \infty$. It will always be required that $T_{n} \uparrow \infty$ as $n \uparrow \infty$, which will be achieved if $N_{n} \uparrow \infty$ and/or $h_{n} \downarrow 0$. It is then natural to consider the triangular array of random variables $\left\{\left\{y_{n t}\right\}_{t=1}^{T_{n}}\right\}_{n=1}^{\infty}$ generated by

$$
y_{n t}=\alpha_{n} y_{n t-1}+u_{n t}, \quad t=1, \ldots, T_{n},
$$

where $\alpha_{n}=e^{\gamma h_{n}}, y_{n 0}=y(0)$ is fixed, and $u_{n t}$ has variance $\gamma_{0}\left(h_{n}\right)$ and autocovariance $\gamma_{1}\left(h_{n}\right)$, as defined in Theorem 1.

The estimators under consideration are the ordinary least squares (OLS) estimator of $\alpha_{n}$ in (7) and an instrumental variable (IV) estimator defined by

$$
\widehat{\alpha}_{n}=\frac{\sum_{t=1}^{T_{n}} y_{n t-1} y_{n t}}{\sum_{t=1}^{T_{n}} y_{n t-1}^{2}} \text { and } \widetilde{\alpha}_{n}=\frac{\sum_{t=1}^{T_{n}} y_{n t-2} y_{n t}}{\sum_{t=1}^{T_{n}} y_{n t-2} y_{n t-1}}
$$

respectively. The OLS estimator has been shown by Phillips (1987a) to be a consistent estimator of $\alpha_{n}$ under the null hypothesis of $\gamma=0$ but is perhaps not a natural estimator under the alternative here in view of the MA disturbance. In fact, $\widehat{\alpha}_{n}$ is inconsistent under $\gamma<0$ because of the correlation between $y_{n t-1}$ and $u_{n t}$. The IV estimator, in contrast, is a consistent estimator of $\alpha_{n}$ even when $\gamma<0$, and was suggested by Hall (1989) for the purposes of testing for unit roots in ARMA models. The test statistics that will be considered are the normalised estimators

$$
\widehat{\phi}_{n}\left(\widehat{\alpha}_{n}-1\right) \text { and } \widetilde{\phi}_{n}\left(\widetilde{\alpha}_{n}-1\right)
$$

where $\widehat{\phi}_{n}$ and $\widetilde{\phi}_{n}$ denote the appropriate normalisations. The consistency of these statistics will be investigated by deriving the limiting distribution under the null hypothesis $(\gamma=0)$ 
and the alternative $(\gamma \neq 0)$ and then examining whether the power tends to unity as $n \rightarrow \infty$. Three main cases will be considered. The first is where $N_{n}=N$ is fixed and $h_{n} \downarrow 0$; the second is where $N_{n} \uparrow \infty$ and $h_{n} \downarrow 0$; the third is where $N_{n} \uparrow \infty$ and $h_{n}=h$ is fixed. In all cases $T_{n} \uparrow \infty$. In cases where the test statistics (9) may be inconsistent, the scope for developing consistent alternatives will also be explored. In some cases, it is also necessary to introduce further sub-cases to adequately capture the asymptotic properties. These depend on the value of $\gamma$ under the alternative hypothesis and also on the behaviour of $h_{n}^{3} N_{n}$ when considering the IV estimator under the alternative hypothesis. Details are given in section 4 . The principal set of cases and sub-cases used is defined in Table 1 so as to avoid repetition later in the paper.

Table 1

Combinations of $N_{n}, h_{n}$ and $y(0)$

\begin{tabular}{ll}
\hline Case & Description (as $n \uparrow \infty)$ \\
\hline 1 (a) & $N_{n}=N, h_{n} \downarrow 0, y(0)=0$ \\
1 (b) & $N_{n}=N, h_{n} \downarrow 0, y(0) \neq 0$ \\
2 (a) & $N_{n} \uparrow \infty, h_{n} \downarrow 0, y(0)=0$ \\
2 (b) & $N_{n} \uparrow \infty, h_{n} \downarrow 0, y(0) \neq 0$ \\
2 (b)(i) & $N_{n} \uparrow \infty, h_{n} \downarrow 0, h_{n} N_{n}^{1 / 2} \downarrow 0, y(0) \neq 0$ \\
$2(\mathrm{~b})(\mathrm{ii})$ & $N_{n} \uparrow \infty, h_{n} \downarrow 0, h_{n} N_{n}^{1 / 2} \rightarrow k, y(0) \neq 0$ \\
$2(\mathrm{~b})(\mathrm{iii})$ & $N_{n} \uparrow \infty, h_{n} \downarrow 0, h_{n} N_{n}^{1 / 2} \uparrow \infty, y(0) \neq 0$ \\
3 & $N_{n} \uparrow \infty, h_{n}=h$ \\
$3(\mathrm{a})$ & $N_{n} \uparrow \infty, h_{n}=h, y(0)=0$ \\
$3(\mathrm{~b})$ & $N_{n} \uparrow \infty, h_{n}=h, y(0) \neq 0$
\end{tabular}

Note: The constants $N, k$ and $h$ are all positive.

\section{The distributions under the null hypothesis}

Under $\gamma=0, y_{n t}$ evolves according to the random walk

$$
y_{n t}=y_{n t-1}+u_{n t}, \quad y_{n 0}=y(0), \quad t=1, \ldots, T_{n}=N_{n} / h_{n},
$$

where, from Theorem $1(\mathrm{~b}), u_{n t}$ is an MA(1) process with variance $(2 / 3) \sigma^{2} h_{n}^{3}$ and autocovariance $(1 / 6) \sigma^{2} h_{n}^{3}$. When $h_{n} \downarrow 0$ as $n \uparrow \infty$, the variance of $u_{n t}$ tends to zero, and so for the asymptotic analysis in such cases the normalised disturbances $u_{n t}^{h}=u_{n t} / h_{n}^{3 / 2}$ are 
considered. Since $u_{n t}$ is Gaussian it follows that $u_{n t}^{h}$ is also a Gaussian MA(1) process with variance $(2 / 3) \sigma^{2}$ and autocovariance $(1 / 6) \sigma^{2}$. As a consequence, the following weak laws of large numbers apply as $n \uparrow \infty$ :

$$
\frac{1}{T_{n}} \sum_{t=1}^{T_{n}} u_{n t}^{h} \stackrel{p}{\rightarrow} 0, \quad \frac{1}{T_{n}} \sum_{t=1}^{T_{n}}\left(u_{n t}^{h}\right)^{2} \stackrel{p}{\rightarrow} \frac{2}{3} \sigma^{2}, \quad \text { and } \frac{1}{T_{n}} \sum_{t=1}^{T_{n}} u_{n t}^{h} u_{n t-1}^{h} \stackrel{p}{\rightarrow} \frac{1}{6} \sigma^{2},
$$

where $\stackrel{p}{\rightarrow}$ denotes convergence in probability. Furthermore, an invariance principle applies to the partial sum process $S_{n t}=\sum_{j=1}^{t} u_{n j}$, so that the random function

$$
X_{n}(r)=T_{n}^{-1 / 2} h_{n}^{-3 / 2} S_{n\left[T_{n} r\right]}=T_{n}^{-1 / 2} h_{n}^{-3 / 2} \sum_{j=1}^{\left[T_{n} r\right]} u_{n j} \Rightarrow \sigma W(r)
$$

as $n \uparrow \infty$, where $[x]$ denotes the integer part of $x, \Rightarrow$ denotes weak convergence of the associated probability measures, and $W(r)$ is a standard Wiener process on $r \in[0,1] .{ }^{2}$ The validity of this invariance principle is easily demonstrated by checking that the conditions of, say, Lemma 2.2 of Phillips (1987a) are satisfied. Since, when $\gamma=0$,

$$
\widehat{\alpha}_{n}-1=\frac{\sum_{t=1}^{T_{n}} y_{n t-1} u_{n t}}{\sum_{t=1}^{T_{n}} y_{n t-1}^{2}} \text { and } \widetilde{\alpha}_{n}-1=\frac{\sum_{t=1}^{T_{n}} y_{n t-2} u_{n t}}{\sum_{t=1}^{T_{n}} y_{n t-2} y_{n t-1}},
$$

the above convergence results are used to derive the properties of the sample moments $\sum_{t=1}^{T_{n}} y_{n t-1} u_{n t}, \sum_{t=1}^{T_{n}} y_{n t-1}^{2}, \sum_{t=1}^{T_{n}} y_{n t-2} u_{n t}$ and $\sum_{t=1}^{T_{n}} y_{n t-2} y_{n t-1}$. The limiting properties of the first two can be used to derive the properties of the latter two using the relationships

$$
\sum_{t=1}^{T_{n}} y_{n t-2} u_{n t}=\sum_{t=1}^{T_{n}} y_{n t-1} u_{n t}-\sum_{t=1}^{T_{n}} u_{n t} u_{n t-1}
$$

which is obtained by substituting $y_{n t-2}=y_{n t-1}-u_{n t-1}$, and

$$
\sum_{t=1}^{T_{n}} y_{n t-2} y_{n t-1}=\sum_{t=1}^{T_{n}} y_{n t-2}^{2}+\sum_{t=1}^{T_{n}} y_{n t-2} u_{n t-1},
$$

obtained by the substitution $y_{n t-1}=y_{n t-2}+u_{n t-1}$. The results of interest are given in Lemma 1 below. It is convenient, for presentation of the results, to define the random variables

$$
\Phi(W, \omega)=\frac{\sigma^{2}}{2}\left[W(1)^{2}-\omega\right], \quad \Psi(W)=\sigma \int_{0}^{1} W(r) d r \text { and } \Psi\left(W^{2}\right)=\sigma^{2} \int_{0}^{1} W(r)^{2} d r,
$$

where $\omega$ is a constant. These random variables occur frequently in the limits in Lemma 1.

\footnotetext{
${ }^{2}$ Note that the long run variance here is $(2 / 3) \sigma^{2}+2(1 / 6) \sigma^{2}=\sigma^{2}$.
} 
Lemma 1. Let $\left\{\left\{y_{n t}\right\}_{t=1}^{T_{n}}\right\}_{n=1}^{\infty}$ denote a triangular array of random variables generated by (10). Then, under the null hypothesis $\gamma=0$, as $n \uparrow \infty$ :

Case 1(a): $\quad N_{n}=N, h_{n} \downarrow 0, y(0)=0$.

$$
\begin{aligned}
& \frac{1}{h_{n}^{2}} \sum_{t=1}^{T_{n}} y_{n t-1} u_{n t} \Rightarrow N \Phi(W, 2 / 3), \quad \frac{1}{h_{n}} \sum_{t=1}^{T_{n}} y_{n t-1}^{2} \Rightarrow N^{2} \Psi\left(W^{2}\right), \\
& \frac{1}{h_{n}^{2}} \sum_{t=1}^{T_{n}} y_{n t-2} u_{n t} \Rightarrow N \Phi(W, 1), \quad \frac{1}{h_{n}} \sum_{t=1}^{T_{n}} y_{n t-2} y_{n t-1} \Rightarrow N^{2} \Psi\left(W^{2}\right) .
\end{aligned}
$$

Case 1(b): $\quad N_{n}=N, h_{n} \downarrow 0, y(0) \neq 0$.

$$
\begin{aligned}
& \frac{1}{h_{n}} \sum_{t=1}^{T_{n}} y_{n t-1} u_{n t} \Rightarrow N^{1 / 2} \sigma y(0) W(1), \quad h_{n} \sum_{t=1}^{T_{n}} y_{n t-1}^{2} \stackrel{p}{\rightarrow} N y(0)^{2}, \\
& \frac{1}{h_{n}} \sum_{t=1}^{T_{n}} y_{n t-2} u_{n t} \Rightarrow N^{1 / 2} \sigma y(0) W(1), \quad h_{n} \sum_{t=1}^{T_{n}} y_{n t-2} y_{n t-1} \stackrel{p}{\rightarrow} N y(0)^{2} .
\end{aligned}
$$

Case 2(a): $\quad N_{n} \uparrow \infty, h_{n} \downarrow 0, y(0)=0$.

$$
\begin{array}{ll}
\frac{1}{h_{n}^{2} N_{n}} \sum_{t=1}^{T_{n}} y_{n t-1} u_{n t} \Rightarrow \Phi(W, 2 / 3), & \frac{1}{h_{n} N_{n}^{2}} \sum_{t=1}^{T_{n}} y_{n t-1}^{2} \Rightarrow \Psi\left(W^{2}\right), \\
\frac{1}{h_{n}^{2} N_{n}} \sum_{t=1}^{T_{n}} y_{n t-2} u_{n t} \Rightarrow \Phi(W, 1), & \frac{1}{h_{n} N_{n}^{2}} \sum_{t=1}^{T_{n}} y_{n t-2} y_{n t-1} \Rightarrow \Psi\left(W^{2}\right) .
\end{array}
$$

Case 2(b)(i): $\quad N_{n} \uparrow \infty, h_{n} \downarrow 0, h_{n} N_{n}^{1 / 2} \downarrow 0, y(0) \neq 0$.

$$
\begin{aligned}
& \frac{1}{h_{n} N_{n}^{1 / 2}} \sum_{t=1}^{T_{n}} y_{n t-1} u_{n t} \Rightarrow \sigma y(0) W(1), \quad \frac{h_{n}}{N_{n}} \sum_{t=1}^{T_{n}} y_{n t-1}^{2} \stackrel{p}{\rightarrow} y(0)^{2}, \\
& \frac{1}{h_{n} N_{n}^{1 / 2}} \sum_{t=1}^{T_{n}} y_{n t-2} u_{n t} \Rightarrow \sigma y(0) W(1), \quad \frac{h_{n}}{N_{n}} \sum_{t=1}^{T_{n}} y_{n t-2} y_{n t-1} \stackrel{p}{\rightarrow} y(0)^{2} .
\end{aligned}
$$

Case 2(b)(ii): $\quad N_{n} \uparrow \infty, h_{n} \downarrow 0, h_{n} N_{n}^{1 / 2} \rightarrow k, y(0) \neq 0$.

$$
\begin{aligned}
& \sum_{t=1}^{T_{n}} y_{n t-1} u_{n t} \Rightarrow k^{2} \Phi(W, 2 / 3)+k \sigma y(0) W(1), \\
& \frac{h_{n}}{N_{n}} \sum_{t=1}^{T_{n}} y_{n t-1}^{2} \Rightarrow k^{2} \Psi\left(W^{2}\right)+2 k y(0) \Psi(W)+y(0)^{2}, \\
& \sum_{t=1}^{T_{n}} y_{n t-2} u_{n t} \Rightarrow k^{2} \Phi(W, 1)+k \sigma y(0) W(1), \\
& \frac{h_{n}}{N_{n}} \sum_{t=1}^{T_{n}} y_{n t-2} y_{n t-1} \Rightarrow k^{2} \Psi\left(W^{2}\right)+2 k y(0) \Psi(W)+y(0)^{2} .
\end{aligned}
$$


Case 2(b)(iii): $\quad N_{n} \uparrow \infty, h_{n} \downarrow 0, h_{n} N_{n}^{1 / 2} \uparrow \infty, y(0) \neq 0$.

$$
\begin{array}{ll}
\frac{1}{h_{n}^{2} N_{n}} \sum_{t=1}^{T_{n}} y_{n t-1} u_{n t} \Rightarrow \Phi(W, 2 / 3), & \frac{1}{h_{n} N_{n}^{2}} \sum_{t=1}^{T_{n}} y_{n t-1}^{2} \Rightarrow \Psi\left(W^{2}\right), \\
\frac{1}{h_{n}^{2} N_{n}} \sum_{t=1}^{T_{n}} y_{n t-2} u_{n t} \Rightarrow \Phi(W, 1), & \frac{1}{h_{n} N_{n}^{2}} \sum_{t=1}^{T_{n}} y_{n t-2} y_{n t-1} \Rightarrow \Psi\left(W^{2}\right) .
\end{array}
$$

Case 3: $N_{n} \uparrow \infty, h_{n}=h$.

$$
\begin{aligned}
& \frac{1}{T_{n}} \sum_{t=1}^{T_{n}} y_{n t-1} u_{n t} \Rightarrow h^{3} \Phi(W, 2 / 3), \quad \frac{1}{T_{n}^{2}} \sum_{t=1}^{T_{n}} y_{n t-1}^{2} \Rightarrow h^{3} \Psi\left(W^{2}\right), \\
& \frac{1}{T_{n}} \sum_{t=1}^{T_{n}} y_{n t-2} u_{n t} \Rightarrow h^{3} \Phi(W, 1), \quad \frac{1}{T_{n}^{2}} \sum_{t=1}^{T_{n}} y_{n t-2} y_{n t-1} \Rightarrow h^{3} \Psi\left(W^{2}\right) .
\end{aligned}
$$

A number of features of Lemma 1 are worth commenting upon. First, it is apparent that different rates of convergence apply in the different cases and, with the exception of case 3 , that the limiting behaviour depends on whether $y(0)$ is zero or not. The results in case 3 correspond to the usual asymptotics obtained in unit root autoregression with a fixed sampling frequency and an MA(1) disturbance, and are covered by Theorem 3.1 of Phillips (1987a). In this case, the effect of the initial condition vanishes asymptotically. Lemma 1 also establishes that in case 2(b) the relative rates at which $h_{n} \downarrow 0$ and $N_{n} \uparrow \infty$ are important when $y(0) \neq 0$. Three possible types of behaviour for the product $h_{n} N_{n}^{1 / 2}$ are considered and the limits of the sample moments, and the appropriate normalisations, are different in each case. This feature does not occur with stock data, in which the relative rates of convergence of $h_{n}$ and $N_{n}$ to their limits is not important; see Lemma 1 of Perron (1991).

The limiting distributions of the appropriately normalised estimators $\widehat{\alpha}_{n}$ and $\widetilde{\alpha}_{n}$ are given in Theorem 2, in which the notation $Z(W, \omega)=\Phi(W, \omega) / \Psi\left(W^{2}\right)$ is used to depict a commonly-occuring random variable. Note that the variable $Z(W, 1)$ denotes the familiar limiting distribution of $T_{n}\left(\widehat{\alpha}_{n}-1\right)$ when $h=1$ and $u_{n t}$ is a white noise process; see equation (10) of Phillips (1987a).

Theorem 2. Let $\left\{\left\{y_{n t}\right\}_{t=1}^{T_{n}}\right\}_{n=1}^{\infty}$ denote a triangular array of random variables generated by (10). Then, under the null hypothesis $\gamma=0$, as $n \uparrow \infty$ :

Case 1(a): $\quad N_{n}=N, h_{n} \downarrow 0, y(0)=0$.

$$
T_{n}\left(\widehat{\alpha}_{n}-1\right) \Rightarrow Z(W, 2 / 3), \quad T_{n}\left(\widetilde{\alpha}_{n}-1\right) \Rightarrow Z(W, 1) .
$$


Case 1(b): $N_{n}=N, h_{n} \downarrow 0, y(0) \neq 0$.

$$
T_{n}^{2}\left(\widehat{\alpha}_{n}-1\right) \Rightarrow \frac{\sigma N^{3 / 2} W(1)}{y(0)}, \quad T_{n}^{2}\left(\widetilde{\alpha}_{n}-1\right) \Rightarrow \frac{\sigma N^{3 / 2} W(1)}{y(0)} .
$$

Case 2(a): $N_{n} \uparrow \infty, h_{n} \downarrow 0, y(0)=0$.

$$
T_{n}\left(\widehat{\alpha}_{n}-1\right) \Rightarrow Z(W, 2 / 3), \quad T_{n}\left(\widetilde{\alpha}_{n}-1\right) \Rightarrow Z(W, 1) .
$$

Case 2(b)(i): $\quad N_{n} \uparrow \infty, h_{n} \downarrow 0, h_{n} N_{n}^{1 / 2} \downarrow 0, y(0) \neq 0$.

$$
\frac{T_{n}^{2}}{N_{n}^{3 / 2}}\left(\widehat{\alpha}_{n}-1\right) \Rightarrow \frac{\sigma W(1)}{y(0)}, \quad \frac{T_{n}^{2}}{N_{n}^{3 / 2}}\left(\widetilde{\alpha}_{n}-1\right) \Rightarrow \frac{\sigma W(1)}{y(0)} .
$$

Case 2(b)(ii): $\quad N_{n} \uparrow \infty, h_{n} \downarrow 0, h_{n} N_{n}^{1 / 2} \rightarrow k, y(0) \neq 0$.

$$
\begin{aligned}
& T_{n}\left(\widehat{\alpha}_{n}-1\right) \Rightarrow \frac{k^{2} \Phi(W, 2 / 3)+k \sigma y(0) W(1)}{k^{2} \Psi\left(W^{2}\right)+2 k y(0) \Psi(W)+y(0)^{2}}, \\
& T_{n}\left(\widetilde{\alpha}_{n}-1\right) \Rightarrow \frac{k^{2} \Phi(W, 1)+k \sigma y(0) W(1)}{k^{2} \Psi\left(W^{2}\right)+2 k y(0) \Psi(W)+y(0)^{2}} .
\end{aligned}
$$

Case 2(b)(iii): $\quad N_{n} \uparrow \infty, h_{n} \downarrow 0, h_{n} N_{n}^{1 / 2} \uparrow \infty, y(0) \neq 0$.

$$
T_{n}\left(\widehat{\alpha}_{n}-1\right) \Rightarrow Z(W, 2 / 3), \quad T_{n}\left(\widetilde{\alpha}_{n}-1\right) \Rightarrow Z(W, 1) .
$$

Case 3: $N_{n} \uparrow \infty, h_{n}=h$.

$$
T_{n}\left(\widehat{\alpha}_{n}-1\right) \Rightarrow Z(W, 2 / 3), \quad T_{n}\left(\widetilde{\alpha}_{n}-1\right) \Rightarrow Z(W, 1) .
$$

It is worthwhile commenting on the results in Theorem 2 on a case-by-case basis. In case 1 , $T_{n}\left(\widehat{\alpha}_{n}-1\right)$ converges to the random variable $Z(W, 2 / 3)$ when $y(0)=0$, which written more fully gives

$$
T_{n}\left(\widehat{\alpha}_{n}-1\right) \Rightarrow \frac{\frac{1}{2}\left[W(1)^{2}-\frac{2}{3}\right]}{\int_{0}^{1} W(r)^{2} d r}
$$

as $n \uparrow \infty$. The adjustment factor of $2 / 3$ in the numerator arises due to the correlation between the regressor $y_{n t-1}$ and the disturbance $u_{n t}$, and is equal to the ratio of the variance of $u_{n t} / h_{n}^{3 / 2}$, equal to $2 \sigma^{2} / 3$, to the long run variance of the normalised partial sum of $u_{n t} / h_{n}^{3 / 2}, X_{n}(r)$, which equals $\sigma^{2}$. This is precisely the distribution that would be expected from Theorem 3.1 of Phillips (1987a) when $h=1$ and $T_{n} \uparrow \infty$, and indeed occurs in case 3. The IV estimator adjusts for the correlation between the regressor and the disturbance, 
resulting in the familiar distribution $Z(W, 1)$. When $y(0) \neq 0$, the initial value is important, and it turns out that the appropriate normalisation for $\widehat{\alpha}_{n}-1$ and $\widetilde{\alpha}_{n}-1$ is $T_{n}^{2}$, rather than $T_{n}$, the limiting distibution being $N\left(0, \sigma^{2} N^{3} / y(0)^{2}\right)$ in this case since $W(1) \sim N(0,1)$. In fact, $T_{n}\left(\widehat{\alpha}_{n}-1\right)$ and $T_{n}\left(\widetilde{\alpha}_{n}-1\right)$ both converge to zero in this case. In case 2 , the limiting distribution of the normalised OLS estimator $\widehat{\alpha}_{n}$ is once more $Z(W, 2 / 3)$ in parts (a) and (b)(iii), in the latter case the condition $h_{n} N_{n}^{1 / 2} \uparrow \infty$ ensuring that the effects of the initial condition disappear asymptotically. The same holds for the IV estimator $\widetilde{\alpha}_{n}$, except that the limiting distribution is $Z(W, 1)$. In parts (b)(i) and (b)(ii) of case 2 , different behaviour of $h_{n} N_{n}^{1 / 2}$ as $n \uparrow \infty$ results in different limiting distributions, in part (b)(i) the appropriate normalisation being $T_{n}^{2} / N_{n}^{3 / 2}$ for both estimators. In part (b)(i) the limiting distributions are once again normal in view of $W(1)$ being a standard normal random variable.

\section{The distributions under the alternative hypothesis}

When $\gamma \neq 0, y_{n t}$ evolves according to (7) i.e. $y_{n t}=\alpha_{n} y_{n t-1}+u_{n t},\left(t=1, \ldots, T_{n}\right)$, where $\alpha_{n}=e^{\gamma h_{n}}$. In this case, interest centres on

$$
\widehat{\alpha}_{n}-\alpha_{n}=\frac{\sum_{t=1}^{T_{n}} y_{n t-1} u_{n t}}{\sum_{t=1}^{T_{n}} y_{n t-1}^{2}} \text { and } \widetilde{\alpha}_{n}-\alpha_{n}=\frac{\sum_{t=1}^{T_{n}} y_{n t-2} u_{n t}}{\sum_{t=1}^{T_{n}} y_{n t-2} y_{n t-1}},
$$

and Lemma 2 below provides the limiting properties of suitably normalised versions of the numerators and denominators. As in section 3, the sample moments that define the IV estimator can be derived from those defining the OLS estimator via the following formulae:

$$
\begin{aligned}
& \sum_{t=1}^{T_{n}} y_{n t-2} u_{n t}=e^{-\gamma h_{n}} \sum_{t=1}^{T_{n}} y_{n t-1} u_{n t}-e^{-\gamma h_{n}} \sum_{t=1}^{T_{n}} u_{n t} u_{n t-1}, \\
& \sum_{t=1}^{T_{n}} y_{n t-2} y_{n t-1}=e^{\gamma h_{n}} \sum_{t=1}^{T_{n}} y_{n t-2}^{2}+\sum_{t=1}^{T_{n}} y_{n t-2} u_{n t-1} .
\end{aligned}
$$

Note that in case 3 , when $h_{n}=h$ is fixed, the true value of $\alpha_{n}=\alpha=e^{\gamma h}$, which is independent of $n$. When $h_{n} \downarrow 0$ as $n \uparrow \infty$, the laws of large numbers in (11) apply, but when $h_{n}=h$ is fixed,

$$
\frac{1}{T_{n}} \sum_{t=1}^{T_{n}} u_{n t} \stackrel{p}{\rightarrow} 0, \quad \frac{1}{T_{n}} \sum_{t=1}^{T_{n}} u_{n t}^{2} \stackrel{p}{\rightarrow} \gamma_{0}(h), \quad \text { and } \quad \frac{1}{T_{n}} \sum_{t=1}^{T_{n}} u_{n t} u_{n t-1} \stackrel{p}{\rightarrow} \gamma_{1}(h)
$$


where the functions $\gamma_{0}(h)$ and $\gamma_{1}(h)$ are defined in Theorem 1(a). The characterisation of the limits in Lemma 2 is aided by defining the stochastic integral

$$
J(r)=\int_{0}^{r} e^{(r-s) \gamma N} d W(s), \quad 0 \leq r \leq 1
$$

For fixed $r, J(r) \sim N\left(0,\left(e^{2 r \gamma N}-1\right) / 2 \gamma N\right)$. It is also convenient to define the random variables

$$
\Psi\left(J^{2}\right)=\sigma^{2} \int_{0}^{1} J(r)^{2} d r \text { and } \Omega(J, W, \omega)=\frac{\sigma^{2}}{2}\left[\int_{0}^{1} J(r) d W(r)+\omega\right]
$$

for some constant $\omega$, as well as two independent standard normal random variables, denoted $\xi$ and $\eta$. Finally, the constant

$$
\theta_{h}=\frac{\alpha^{2} \gamma_{0}(h)+2 \alpha \gamma_{1}(h)}{\alpha^{2}-1}=\frac{e^{2 \gamma h} \gamma_{0}(h)+2 e^{\gamma h} \gamma_{1}(h)}{e^{2 \gamma h}-1}
$$

is used as a scaling factor, and $\stackrel{d}{\rightarrow}$ denotes convergence in distribution.

Lemma 2. Let $\left\{\left\{y_{n t}\right\}_{t=1}^{T_{n}}\right\}_{n=1}^{\infty}$ denote a triangular array of random variables generated by (7). Then, under the alternative hypothesis $\gamma \neq 0$, as $n \uparrow \infty$ :

Case 1(a): $\quad N_{n}=N, h_{n} \downarrow 0, y(0)=0$.

$$
\begin{aligned}
& \frac{1}{h_{n}^{2}} \sum_{t=1}^{T_{n}} y_{n t-1} u_{n t} \Rightarrow N \Omega(J, W, 1 / 6), \quad \frac{1}{h_{n}} \sum_{t=1}^{T_{n}} y_{n t-1}^{2} \Rightarrow N^{2} \Psi\left(J^{2}\right), \\
& \frac{1}{h_{n}^{2}} \sum_{t=1}^{T_{n}} y_{n t-2} u_{n t} \Rightarrow N \Omega(J, W, 0), \quad \frac{1}{h_{n}} \sum_{t=1}^{T_{n}} y_{n t-2} y_{n t-1} \Rightarrow N^{2} \Psi\left(J^{2}\right) .
\end{aligned}
$$

Case 1(b): $\quad N_{n}=N, h_{n} \downarrow 0, y(0) \neq 0$.

$$
\begin{aligned}
& \sum_{t=1}^{T_{n}} y_{n t-1} u_{n t} \stackrel{p}{\rightarrow} 0, \quad h_{n} \sum_{t=1}^{T_{n}} y_{n t-1}^{2} \Rightarrow y(0)^{2} \frac{\left(e^{2 \gamma N}-1\right)}{2 \gamma} \\
& \sum_{t=1}^{T_{n}} y_{n t-2} u_{n t} \stackrel{p}{\rightarrow} 0, \quad h_{n} \sum_{t=1}^{T_{n}} y_{n t-2} y_{n t-1} \Rightarrow y(0)^{2} \frac{\left(e^{2 \gamma N}-1\right)}{2 \gamma} .
\end{aligned}
$$


Case 2(a): $\quad N_{n} \uparrow \infty, h_{n} \downarrow 0, y(0)=0$.

$$
\begin{aligned}
& \text { If } \gamma>0: \quad \frac{2 \gamma N_{n}}{e^{\gamma N_{n}} h_{n}^{2} N_{n}} \sum_{t=1}^{T_{n}} y_{n t-1} u_{n t} \Rightarrow \sigma^{2} \xi \eta, \quad \frac{\left(2 \gamma N_{n}\right)^{2}}{e^{2 \gamma N_{n}} h_{n} N_{n}^{2}} \sum_{t=1}^{T_{n}} y_{n t-1}^{2} \Rightarrow \sigma^{2} \eta^{2}, \\
& \frac{2 \gamma N_{n}}{e^{\gamma N_{n}} h_{n}^{2} N_{n}} \sum_{t=1}^{T_{n}} y_{n t-2} u_{n t} \Rightarrow \sigma^{2} \xi \eta, \quad \frac{\left(2 \gamma N_{n}\right)^{2}}{e^{2 \gamma N_{n}} h_{n} N_{n}^{2}} \sum_{t=1}^{T_{n}} y_{n t-2} y_{n t-1} \Rightarrow \sigma^{2} \eta^{2} . \\
& \text { If } \gamma<0: \quad \frac{\left(-2 \gamma N_{n}\right)^{1 / 2}}{h_{n}^{2} N_{n}} \sum_{t=1}^{T_{n}} y_{n t-1} u_{n t} \Rightarrow \sigma^{2} N(0,1), \quad \frac{-2 \gamma N_{n}}{h_{n} N_{n}^{2}} \sum_{t=1}^{T_{n}} y_{n t-1}^{2} \stackrel{p}{\rightarrow} \sigma^{2} \text {, } \\
& \frac{1}{h_{n}^{2} N_{n}} \sum_{t=1}^{T_{n}} y_{n t-2} u_{n t} \stackrel{p}{\rightarrow}-\frac{1}{6} \sigma^{2}, \quad \frac{-2 \gamma N_{n}}{h_{n} N_{n}^{2}} \sum_{t=1}^{T_{n}} y_{n t-2} y_{n t-1} \stackrel{p}{\rightarrow} \sigma^{2} .
\end{aligned}
$$

Case 2(b): $\quad N_{n} \uparrow \infty, h_{n} \downarrow 0, y(0) \neq 0$.

$$
\begin{aligned}
\text { If } \gamma>0: \quad & \frac{1}{e^{2 \gamma N_{n}}} \sum_{t=1}^{T_{n}} y_{n t-1} u_{n t} \stackrel{p}{\rightarrow} 0, \quad \frac{h_{n}}{e^{2 \gamma N_{n}}} \sum_{t=1}^{T_{n}} y_{n t-1}^{2} \stackrel{p}{\rightarrow} \frac{y(0)^{2}}{2 \gamma}, \\
& \frac{1}{e^{2 \gamma N_{n}}} \sum_{t=1}^{T_{n}} y_{n t-2} u_{n t} \stackrel{p}{\rightarrow} 0, \quad \frac{h_{n}}{e^{2 \gamma N_{n}}} \sum_{t=1}^{T_{n}} y_{n t-2} y_{n t-1} \stackrel{p}{\rightarrow} \frac{y(0)^{2}}{2 \gamma} .
\end{aligned}
$$

Case 2(b)(i): $\quad N_{n} \uparrow \infty, h_{n} \downarrow 0, h_{n} N_{n}^{1 / 2} \downarrow 0, y(0) \neq 0$.

$$
\begin{aligned}
\text { If } \gamma<0: \quad & \sum_{t=1}^{T_{n}} y_{n t-1} u_{n t} \stackrel{p}{\rightarrow} 0, \quad h_{n} \sum_{t=1}^{T_{n}} y_{n t-1}^{2} \stackrel{p}{\rightarrow}-\frac{y(0)^{2}}{2 \gamma}, \\
& \sum_{t=1}^{T_{n}} y_{n t-2} u_{n t} \stackrel{p}{\rightarrow} 0, \quad h_{n} \sum_{t=1}^{T_{n}} y_{n t-2} y_{n t-1} \stackrel{p}{\rightarrow}-\frac{y(0)^{2}}{2 \gamma} .
\end{aligned}
$$

Case 2(b)(ii): $\quad N_{n} \uparrow \infty, h_{n} \downarrow 0, h_{n} N_{n}^{1 / 2} \rightarrow k, y(0) \neq 0$.

$$
\text { If } \gamma<0: \quad \begin{aligned}
T_{n} & \sum_{t=1} y_{n t-1} u_{n t} \stackrel{p}{\rightarrow}-\frac{2 k^{2} \sigma^{2}}{3}, \quad h_{n} \sum_{t=1}^{T_{n}} y_{n t-1}^{2} \stackrel{p}{\rightarrow}-\frac{y(0)^{2}}{2 \gamma}, \\
& \sum_{t=1}^{T_{n}} y_{n t-2} u_{n t} \stackrel{p}{\rightarrow}-\frac{5 k^{2} \sigma^{2}}{6}, \quad h_{n} \sum_{t=1}^{T_{n}} y_{n t-2} y_{n t-1} \stackrel{p}{\rightarrow}-\frac{y(0)^{2}}{2 \gamma} .
\end{aligned}
$$


Case 2(b)(iii): $\quad N_{n} \uparrow \infty, h_{n} \downarrow 0, h_{n} N_{n}^{1 / 2} \uparrow \infty, y(0) \neq 0$.

$$
\begin{aligned}
& \text { If } \gamma<0: \quad \frac{1}{h_{n}^{2} N_{n}} \sum_{t=1}^{T_{n}} y_{n t-1} u_{n t} \stackrel{p}{\rightarrow}-\frac{2 \sigma^{2}}{3}, \quad h_{n} \sum_{t=1}^{T_{n}} y_{n t-1}^{2} \stackrel{p}{\rightarrow}-\frac{y(0)^{2}}{2 \gamma}, \\
& \frac{1}{h_{n}^{2} N_{n}} \sum_{t=1}^{T_{n}} y_{n t-2} u_{n t} \stackrel{p}{\rightarrow}-\frac{5 \sigma^{2}}{6}, \\
& h_{n} \sum_{t=1}^{T_{n}} y_{n t-2} y_{n t-1} \stackrel{p}{\rightarrow}-\frac{y(0)^{2}}{2 \gamma} \text { if } h_{n}^{3} N_{n} \downarrow 0, \\
& h_{n} \sum_{t=1}^{T_{n}} y_{n t-2} y_{n t-1} \stackrel{p}{\rightarrow}-\frac{y(0)^{2}}{2 \gamma}-\frac{5 k^{\prime} \sigma^{2}}{6} \text { if } h_{n}^{3} N_{n} \rightarrow k^{\prime}, \\
& \frac{1}{h_{n}^{2} N_{n}} \sum_{t=1}^{T_{n}} y_{n t-2} y_{n t-1} \stackrel{p}{\rightarrow}-\frac{2 \sigma^{2}}{3} \text { if } h_{n}^{3} N_{n} \uparrow \infty .
\end{aligned}
$$

Case 3: $\quad N_{n} \uparrow \infty, h_{n}=h$.

$$
\begin{aligned}
\text { If } \gamma<0: & \frac{1}{T_{n}} \sum_{t=1}^{T_{n}} y_{n t-1} u_{n t} \stackrel{p}{\rightarrow} \gamma_{1}(h), \quad \frac{1}{T_{n}} \sum_{t=1}^{T_{n}} y_{n t-1}^{2} \stackrel{p}{\rightarrow} \frac{\gamma_{0}(h)+2 e^{\gamma h} \gamma_{1}(h)}{\left(1-e^{2 \gamma h}\right)}, \\
& \frac{1}{T_{n}} \sum_{t=1}^{T_{n}} y_{n t-2} u_{n t} \stackrel{p}{\rightarrow} 0, \quad \frac{1}{T_{n}} \sum_{t=1}^{T_{n}} y_{n t-2} y_{n t-1} \stackrel{p}{\rightarrow} \frac{e^{\gamma h} \gamma_{0}(h)+\left(1+e^{2 \gamma h}\right) \gamma_{1}(h)}{\left(1-e^{2 \gamma h}\right)} .
\end{aligned}
$$

Case 3(a): $\quad N_{n} \uparrow \infty, h_{n}=h, y(0)=0$.

$$
\begin{aligned}
\text { If } \gamma>0: & \frac{1}{\alpha^{\left(T_{n}-2\right)}} \sum_{t=1}^{T_{n}} y_{n t-1} u_{n t} \stackrel{d}{\rightarrow} \theta_{h}^{2} \xi \eta, & \frac{\left(\alpha^{2}-1\right)}{\alpha^{2\left(T_{n}-1\right)} \sum_{t=1}^{T_{n}} y_{n t-1}^{2} \stackrel{d}{\rightarrow} \theta_{h}^{2} \eta^{2},} \\
& \frac{1}{\alpha^{\left(T_{n}-2\right)}} \sum_{t=1}^{T_{n}} y_{n t-2} u_{n t} \stackrel{d}{\rightarrow} e^{-\gamma h} \theta_{h}^{2} \xi \eta, & \frac{\left(\alpha^{2}-1\right)}{\alpha^{2\left(T_{n}-1\right)}} \sum_{t=1}^{T_{n}} y_{n t-2} y_{n t-1} \stackrel{d}{\rightarrow} e^{\gamma h} \theta_{h}^{2} \eta^{2} .
\end{aligned}
$$

Case 3(b): $\quad N_{n} \uparrow \infty, h_{n}=h, y(0) \neq 0$.

$$
\begin{aligned}
\text { If } \gamma>0: \quad & \frac{1}{\alpha^{\left(T_{n}-2\right)}} \sum_{t=1}^{T_{n}} y_{n t-1} u_{n t} \stackrel{d}{\rightarrow} \theta_{h} \xi\left(e^{\gamma h} y(0)+\theta_{h} \eta\right), \\
& \frac{\left(\alpha^{2}-1\right)}{\alpha^{2\left(T_{n}-1\right)}} \sum_{t=1}^{T_{n}} y_{n t-1}^{2} \stackrel{d}{\rightarrow}\left(e^{\gamma h} y(0)+\theta_{h} \eta\right)^{2}, \\
& \frac{1}{\alpha^{\left(T_{n}-2\right)}} \sum_{t=1}^{T_{n}} y_{n t-2} u_{n t} \stackrel{d}{\rightarrow} e^{-\gamma h} \theta_{h} \xi\left(e^{\gamma h} y(0)+\theta_{h} \eta\right), \\
& \frac{\left(\alpha^{2}-1\right)}{\alpha^{2\left(T_{n}-1\right)}} \sum_{t=1}^{T_{n}} y_{n t-2} y_{n t-1} \stackrel{d}{\rightarrow} e^{\gamma h}\left(e^{\gamma h} y(0)+\theta_{h} \eta\right)^{2} .
\end{aligned}
$$

A number of features of the convergence of the sample moments in Lemma 2 are worth commenting upon. In case 1 , with $N$ fixed and $h_{n} \downarrow 0$, the convergence does not depend on whether $\gamma$ is in the explosive or stable region, but more on the value of $y(0)$. This is not 
so in cases 2 and 3, where rather different limiting behaviour is obtained in the stable and explosive cases. In cases 2 and 3, the effects of $y(0)$ are also important. In case 2(b)(iii), when $\gamma<0$, the behaviour of $h_{n}^{3} N_{n}$ as $n \uparrow \infty$ is important and leads to different normalisations (and different limits) for $\sum_{t=1}^{T_{n}} y_{n t-2} y_{n t-1}$, depending on whether $h_{n}^{3} N_{n} \downarrow 0, \rightarrow k^{\prime}$ or $\uparrow \infty$. The results in cases 3(a) and 3(b), in which $\gamma>0$ and hence $y_{n t}$ is explosive, are derived by extending the methods of Anderson (1959) to the case of an $\operatorname{ARMA}(1,1)$ process.

The results in Lemma 2 enable the limiting distributions of the appropriately normalised differences $\left(\widehat{\alpha}_{n}-\alpha_{n}\right)$ and $\left(\widetilde{\alpha}_{n}-\alpha_{n}\right)$ to be obtained. These are presented in Theorem 3 , along with the limiting distributions of the appropriately normalised deviations $\left(\widehat{\alpha}_{n}-1\right)$ and $\left(\widetilde{\alpha}_{n}-1\right)$.

Theorem 3. Let $\left\{\left\{y_{n t}\right\}_{t=1}^{T_{n}}\right\}_{n=1}^{\infty}$ denote a triangular array of random variables generated by (7). Then, under the alternative hypothesis $\gamma \neq 0$, as $n \uparrow \infty$ :

Case 1(a): $\quad N_{n}=N, h_{n} \downarrow 0, y(0)=0$.

$$
\begin{array}{ll}
T_{n}\left(\widehat{\alpha}_{n}-\alpha_{n}\right) \Rightarrow \frac{\Omega(J, W, 1 / 6)}{\Psi\left(J^{2}\right)}, & T_{n}\left(\widehat{\alpha}_{n}-1\right) \Rightarrow \frac{\Omega(J, W, 1 / 6)}{\Psi\left(J^{2}\right)}+\gamma N, \\
T_{n}\left(\widetilde{\alpha}_{n}-\alpha_{n}\right) \Rightarrow \frac{\Omega(J, W, 0)}{\Psi\left(J^{2}\right)}, & T_{n}\left(\widetilde{\alpha}_{n}-1\right) \Rightarrow \frac{\Omega(J, W, 0)}{\Psi\left(J^{2}\right)}+\gamma N .
\end{array}
$$

Case 1(b): $\quad N_{n}=N, h_{n} \downarrow 0, y(0) \neq 0$.

$$
\begin{aligned}
& T_{n}\left(\widehat{\alpha}_{n}-\alpha_{n}\right) \stackrel{p}{\rightarrow} 0, \quad T_{n}\left(\widehat{\alpha}_{n}-1\right) \stackrel{p}{\rightarrow} \gamma N, \\
& T_{n}\left(\widetilde{\alpha}_{n}-\alpha_{n}\right) \stackrel{p}{\rightarrow} 0, \quad T_{n}\left(\widetilde{\alpha}_{n}-1\right) \stackrel{p}{\rightarrow} \gamma N .
\end{aligned}
$$

Case 2(a): $\quad N_{n} \uparrow \infty, h_{n} \downarrow 0, y(0)=0$.

$$
\begin{array}{lll}
\text { If } \gamma>0: & \frac{e^{\gamma N_{n}} T_{n}}{2 \gamma N_{n}}\left(\widehat{\alpha}_{n}-\alpha_{n}\right) \Rightarrow \frac{\xi}{\eta}, & \frac{T_{n}}{N_{n}}\left(\widehat{\alpha}_{n}-1\right) \stackrel{p}{\rightarrow} \gamma, \\
& \frac{e^{\gamma N_{n}} T_{n}}{2 \gamma N_{n}}\left(\widetilde{\alpha}_{n}-\alpha_{n}\right) \Rightarrow \frac{\xi}{\eta}, & \frac{T_{n}}{N_{n}}\left(\widetilde{\alpha}_{n}-1\right) \stackrel{p}{\rightarrow} \gamma . \\
\text { If } \gamma<0: & \frac{T_{n}}{\sqrt{\left(-2 \gamma N_{n}\right)}}\left(\widehat{\alpha}_{n}-\alpha_{n}\right) \Rightarrow N(0,1), & \frac{T_{n}}{N_{n}}\left(\widehat{\alpha}_{n}-1\right) \stackrel{p}{\rightarrow} \gamma, \\
& \frac{T_{n}}{\left(-2 \gamma N_{n}\right)}\left(\widetilde{\alpha}_{n}-\alpha_{n}\right) \Rightarrow-\frac{1}{6}, & \frac{T_{n}}{N_{n}}\left(\widetilde{\alpha}_{n}-1\right) \stackrel{p}{\rightarrow} \frac{4 \gamma}{3} .
\end{array}
$$


Case 2(b): $\quad N_{n} \uparrow \infty, h_{n} \downarrow 0, y(0) \neq 0$.

$$
\begin{aligned}
& \text { If } \gamma>0: \quad \frac{T_{n}}{N_{n}}\left(\widehat{\alpha}_{n}-\alpha_{n}\right) \stackrel{p}{\rightarrow} 0, \quad \frac{T_{n}}{N_{n}}\left(\widehat{\alpha}_{n}-1\right) \stackrel{p}{\rightarrow} \gamma, \\
& \frac{T_{n}}{N_{n}}\left(\widetilde{\alpha}_{n}-\alpha_{n}\right) \stackrel{p}{\rightarrow} 0, \quad \frac{T_{n}}{N_{n}}\left(\widetilde{\alpha}_{n}-1\right) \stackrel{p}{\rightarrow} \gamma .
\end{aligned}
$$

Case 2(b)(i): $\quad N_{n} \uparrow \infty, h_{n} \downarrow 0, h_{n} N_{n}^{1 / 2} \downarrow 0, y(0) \neq 0$.

$$
\begin{array}{ll}
\text { If } \gamma<0: \quad & \frac{T_{n}}{N_{n}}\left(\widehat{\alpha}_{n}-\alpha_{n}\right) \stackrel{p}{\rightarrow} 0, \quad \frac{T_{n}}{N_{n}}\left(\widehat{\alpha}_{n}-1\right) \stackrel{p}{\rightarrow} \gamma, \\
& \frac{T_{n}}{N_{n}}\left(\widetilde{\alpha}_{n}-\alpha_{n}\right) \stackrel{p}{\rightarrow} 0, \quad \frac{T_{n}}{N_{n}}\left(\widetilde{\alpha}_{n}-1\right) \stackrel{p}{\rightarrow} \gamma .
\end{array}
$$

Case 2(b)(ii): $\quad N_{n} \uparrow \infty, h_{n} \downarrow 0, h_{n} N_{n}^{1 / 2} \rightarrow k, y(0) \neq 0$.

$$
\begin{aligned}
& \text { If } \gamma<0: \quad \frac{T_{n}}{N_{n}}\left(\widehat{\alpha}_{n}-\alpha_{n}\right) \stackrel{p}{\rightarrow} \frac{4 k^{2} \gamma \sigma^{2}}{3 y(0)^{2}}, \quad \frac{T_{n}}{N_{n}}\left(\widehat{\alpha}_{n}-1\right) \stackrel{p}{\rightarrow} \frac{\gamma\left[4 k^{2} \sigma^{2}+3 y(0)^{2}\right]}{3 y(0)^{2}} \text {, } \\
& \frac{T_{n}}{N_{n}}\left(\widetilde{\alpha}_{n}-\alpha_{n}\right) \stackrel{p}{\rightarrow} \frac{5 k^{2} \gamma \sigma^{2}}{3 y(0)^{2}}, \quad \frac{T_{n}}{N_{n}}\left(\widetilde{\alpha}_{n}-1\right) \stackrel{p}{\rightarrow} \frac{\gamma\left[5 k^{2} \sigma^{2}+3 y(0)^{2}\right]}{3 y(0)^{2}} .
\end{aligned}
$$

Case 2(b)(iii): $\quad N_{n} \uparrow \infty, h_{n} \downarrow 0, h_{n} N_{n}^{1 / 2} \uparrow \infty, y(0) \neq 0$.

$$
\begin{aligned}
& \text { If } \gamma<0: \quad \frac{T_{n}^{3}}{N_{n}^{4}}\left(\widehat{\alpha}_{n}-\alpha_{n}\right) \stackrel{p}{\rightarrow} \frac{4 \gamma \sigma^{2}}{3 y(0)^{2}}, \quad \frac{T_{n}^{3}}{N_{n}^{4}}\left(\widehat{\alpha}_{n}-1\right) \stackrel{p}{\rightarrow} \frac{4 \gamma \sigma^{2}}{3 y(0)^{2}}, \\
& \frac{T_{n}^{3}}{N_{n}^{4}}\left(\widetilde{\alpha}_{n}-\alpha_{n}\right) \stackrel{p}{\rightarrow} \frac{5 \gamma \sigma^{2}}{3 y(0)^{2}}, \quad \frac{T_{n}^{3}}{N_{n}^{4}}\left(\widetilde{\alpha}_{n}-1\right) \stackrel{p}{\rightarrow} \frac{5 \gamma \sigma^{2}}{3 y(0)^{2}} \quad \text { if } \quad h_{n}^{3} N_{n} \downarrow 0, \\
& \frac{T_{n}^{3}}{N_{n}^{4}}\left(\widetilde{\alpha}_{n}-\alpha_{n}\right) \stackrel{p}{\rightarrow} 1+\frac{5 k^{\prime} \gamma \sigma^{2} T_{n}^{3}}{3 y(0)^{2} \dot{N}_{n}^{4}}\left(\widetilde{\alpha}_{n}-1\right) \stackrel{p}{\rightarrow} 1+\frac{5 k^{\prime} \gamma \sigma^{2}}{3 y(0)^{2}} \text { if } h_{n}^{3} N_{n} \rightarrow k^{\prime}, \\
& \left(\widetilde{\alpha}_{n}-\alpha_{n}\right) \stackrel{p}{\rightarrow} \frac{5}{4}, \quad\left(\widetilde{\alpha}_{n}-1\right) \stackrel{p}{\rightarrow} \frac{5}{4} \quad \text { if } h_{n}^{3} N_{n} \uparrow \infty .
\end{aligned}
$$

Case 3: $\quad N_{n} \uparrow \infty, h_{n}=h$.

$$
\begin{aligned}
& \text { If } \gamma<0: \quad\left(\widehat{\alpha}_{n}-\alpha\right) \stackrel{p}{\rightarrow} \frac{\left(1-e^{2 \gamma h}\right) \gamma_{1}(h)}{\gamma_{0}(h)+2 e^{\gamma h} \gamma_{1}(h)}, \quad\left(\widehat{\alpha}_{n}-1\right) \stackrel{p}{\rightarrow} \frac{\left(1-e^{2 \gamma h}\right) \gamma_{1}(h)}{\gamma_{0}(h)+2 e^{\gamma h} \gamma_{1}(h)}+e^{\gamma h}-1 \text {, } \\
& \left(\widetilde{\alpha}_{n}-\alpha\right) \stackrel{p}{\rightarrow} 0, \quad\left(\widetilde{\alpha}_{n}-1\right) \stackrel{p}{\rightarrow} e^{\gamma h}-1 .
\end{aligned}
$$

Case 3(a): $\quad N_{n} \uparrow \infty, h_{n}=h, y(0)=0$.

$$
\begin{array}{rr}
\text { If } \gamma>0: & \frac{\alpha^{T_{n}}}{\alpha^{2}-1}\left(\widehat{\alpha}_{n}-\alpha\right) \stackrel{d}{\rightarrow} \frac{\xi}{\eta}, \quad\left(\widehat{\alpha}_{n}-1\right) \stackrel{p}{\rightarrow} e^{\gamma h}-1, \\
& \frac{\alpha^{T_{n}}}{\alpha^{2}-1}\left(\widetilde{\alpha}_{n}-\alpha\right) \stackrel{d}{\rightarrow} \frac{e^{-2 \gamma h} \xi}{\eta}, \quad\left(\widetilde{\alpha}_{n}-1\right) \stackrel{p}{\rightarrow} e^{\gamma h}-1 .
\end{array}
$$


Case 3(b): $\quad N_{n} \uparrow \infty, h_{n}=h, y(0) \neq 0$.

$$
\begin{aligned}
\text { If } \gamma>0: \quad \frac{\alpha^{T_{n}}}{\alpha^{2}-1}\left(\widehat{\alpha}_{n}-\alpha\right) \stackrel{d}{\rightarrow} \frac{\theta_{h} \xi}{\alpha y(0)+\theta_{h} \eta}=\frac{N\left(0, \theta_{h}^{2}\right)}{N\left(\alpha y(0), \theta_{h}^{2}\right)}, \quad\left(\widehat{\alpha}_{n}-1\right) \stackrel{p}{\rightarrow} e^{\gamma h}-1, \\
\frac{\alpha^{T_{n}}}{\alpha^{2}-1}\left(\widetilde{\alpha}_{n}-\alpha\right) \stackrel{d}{\rightarrow} \frac{e^{-2 \gamma h} \theta_{h} \xi}{\alpha y(0)+\theta_{h} \eta}=\frac{N\left(0, e^{-4 \gamma h} \theta_{h}^{2}\right)}{N\left(\alpha y(0), \theta_{h}^{2}\right)}, \quad\left(\tilde{\alpha}_{n}-1\right) \stackrel{p}{\rightarrow} e^{\gamma h}-1 .
\end{aligned}
$$

The limiting distributions in Theorem 3 are derived from the convergence results in Lemma 2. In case 1(a) the limiting distributions are represented as a functional of the random process $J(r)$, while the results for case 2 , in which $N_{n} \uparrow \infty$, are based on the behaviour of the appropriate functional when $\gamma N_{n} \rightarrow \pm \infty$. It is interesting to note that, in case 2(a), when $\gamma<0$ the appropriate normalisation factor is different for the OLS and IV estimators. The source of this can be traced to Lemma 2 in which the normalisations for $\sum_{t=1}^{T_{n}} y_{n t-1} u_{n t}$ and $\sum_{t=1}^{T_{n}} y_{n t-2} u_{n t}$ differ by a factor of $\left(-2 \gamma N_{n}\right)^{1 / 2}$. The distributions in case $2($ a) when $\gamma>0$ are Cauchy in view of $\xi$ and $\eta$ being independent standard normal random variables. The same is true in case 3(a) when $\gamma>0$. In case 3, when $\gamma<0$ the limiting distributions have not strictly been derived, but for the purposes of establishing the consistency (or otherwise) of the test statistics, the reported probability limits turn out to be sufficient. It is in the analysis of test consistency that the results of Theorems 2 and 3 will now be employed.

\section{The consistency of the test statistics}

A test is consistent if its power tends to unity asymptotically for fixed alternatives, where the power is the probability of correctly rejecting a false null hypothesis. Thus, if $T_{n}\left(\widehat{\alpha}_{n}-1\right)$ is the statistic of interest, and $\kappa$ denotes the critical value of the test, consistency requires that, under the alternative hypothesis,

$$
\lim _{n \uparrow \infty} \operatorname{Pr}\left(T_{n}\left(\widehat{\alpha}_{n}-1\right)>\kappa\right)=1 .
$$

The consistency properties of the test statistics of interest are summarised in Theorem 4 .

Theorem 4. Let $\left\{\left\{y_{n t}\right\}_{t=1}^{T_{n}}\right\}_{n=1}^{\infty}$ denote a triangular array of random variables generated by (7), and consider testing the null hypothesis $\gamma=0$ against a fixed alternative $\gamma \neq 0$. Then: 
Case 1(a): $\quad N_{n}=N, h_{n} \downarrow 0, y(0)=0$.

$T_{n}\left(\widehat{\alpha}_{n}-1\right)$ and $T_{n}\left(\widetilde{\alpha}_{n}-1\right)$ are not consistent.

Case 1(b): $\quad N_{n}=N, h_{n} \downarrow 0, y(0) \neq 0$.

$T_{n}^{2}\left(\widehat{\alpha}_{n}-1\right)$ and $T_{n}^{2}\left(\widetilde{\alpha}_{n}-1\right)$ are consistent.

Case 2(a): $\quad N_{n} \uparrow \infty, h_{n} \downarrow 0, y(0)=0$.

$T_{n}\left(\widehat{\alpha}_{n}-1\right)$ and $T_{n}\left(\widetilde{\alpha}_{n}-1\right)$ are consistent.

Case 2(b)(i): $\quad N_{n} \uparrow \infty, h_{n} \downarrow 0, h_{n} N_{n}^{1 / 2} \downarrow 0, y(0) \neq 0$.

$\left(T_{n}^{2} / N_{n}^{3 / 2}\right)\left(\widehat{\alpha}_{n}-1\right)$ and $\left(T_{n}^{2} / N_{n}^{3 / 2}\right)\left(\widetilde{\alpha}_{n}-1\right)$ are consistent.

Case 2(b)(ii): $\quad N_{n} \uparrow \infty, h_{n} \downarrow 0, h_{n} N_{n}^{1 / 2} \rightarrow k, y(0) \neq 0$.

$T_{n}\left(\widehat{\alpha}_{n}-1\right)$ and $T_{n}\left(\widetilde{\alpha}_{n}-1\right)$ are consistent.

Case $2(b)\left(\right.$ iii): $\quad N_{n} \uparrow \infty, h_{n} \downarrow 0, h_{n} N_{n}^{1 / 2} \uparrow \infty, y(0) \neq 0$.

$T_{n}\left(\widehat{\alpha}_{n}-1\right)$ and $T_{n}\left(\widetilde{\alpha}_{n}-1\right)$ are consistent except when

$\gamma<0$ and $h_{n}^{3} N_{n} \uparrow \infty$.

Case 3: $\quad N_{n} \uparrow \infty, h_{n}=h$.

$T_{n}\left(\widehat{\alpha}_{n}-1\right)$ and $T_{n}\left(\widetilde{\alpha}_{n}-1\right)$ are consistent.

Theorem 4 shows a mixed pattern of consistency for the statistics across the different cases.

The statistics are not consistent at all when $N$ is fixed and $y(0)=0$ (case 1(a)), although when $y(0) \neq 0$ it is possible to construct consistent tests based on $T_{n}^{2}\left(\widehat{\alpha}_{n}-1\right)$ and $T_{n}^{2}\left(\widetilde{\alpha}_{n}-1\right)$. When $N_{n} \uparrow \infty$ as $n \uparrow \infty$ consistent test statistics do exist except in case 2(b)(iii), when $\gamma<0$, $h_{n} N_{n}^{1 / 2} \uparrow \infty$ and $h_{n}^{3} N_{n} \uparrow \infty$.

The results in Theorem 4 contrast with those of Perron (1991) who showed ${ }^{3}$ (with a stock variable) that the statistic $T_{n}\left(\widehat{\alpha}_{n}-1\right)$ is consistent if and only if the span $\left(N_{n}\right)$ increases with $n$. Theorem 4, case 2(b)(iii), identifies an exception to this rule when the variable of interest is a flow. Furthermore, in case 1(b), Theorem 4 indicates that it is possible to conduct a consistent test of the null hypothesis $\gamma=0$ even when the span of the data is fixed. These findings are summarised in the following proposition.

Proposition 1. Let $\left\{\left\{y_{n t}\right\}_{t=1}^{T_{n}}\right\}_{n=1}^{\infty}$ denote a triangular array of random flow variables generated by (7), and consider testing the null hypothesis $\gamma=0$ against a fixed alternative $\gamma \neq 0$. Then:

(a) the condition that $N_{n} \uparrow \infty$ as $n \uparrow \infty$ is not sufficient to ensure that the test is consistent;

\footnotetext{
${ }^{3}$ See Theorem 1 of Perron (1991).
} 
(b) it is possible, in some circumstances, to construct consistent tests even when $N_{n}=N$ is fixed as $n \uparrow \infty$.

The theoretical results contained in Theorem 4 and summarised in Proposition 1 are investigated using Monte Carlo simulations in section 6, and are used to help explain other recently published simulation results.

\section{Some simulation results}

The finite sample content of the theoretical results can be explored with the aid of simulations. In particular, the power of the unit root test statistics in cases 1(a), 1(b) and 2(b)(i) was simulated for the null hypothesis of a unit root $(\gamma=0)$ against the fixed stationary alternative $\gamma=-0.2$. The series were generated, for each fixed span $N$ and frequency $h$, according to

$$
y_{t}=e^{\gamma h} y_{t-1}+u_{t}, \quad t=1, \ldots, T=N / h
$$

where $y_{0}=y(0)$ takes on a fixed value and $u_{t}$ is a normally distributed MA(1) process with variance $\gamma_{0}(h)$ and first-order autocovariance $\gamma_{1}(h)$ (the underlying variance parameter $\left.\sigma^{2}=1\right)$. A total of 10,000 replications were conducted for each combination of $N$ and $h$ considered, which for cases 1 (a) and 1(b) corresponds to the 24 combinations of $N=16$, 32,64 , and 128 and $h=2,1,1 / 2,1 / 4,1 / 12$ and $1 / 52$. The values of $h$ can be interpreted as corresponding to observations at the biennial, annual, biannual, quarterly, monthly and weekly frequencies, respectively, while the data spans are then interpreted to range between 16 and 128 years. For case 2(b)(i) it is required that $h N^{1 / 2} \downarrow 0$ as $h \downarrow 0$ and $N \uparrow \infty$, so the following $(N, h)$ pairs were considered: $(10,2),(12,1),(16,1 / 2),(18,1 / 4),(36,1 / 12)$ and $(240,1 / 52)$. With these combinations $h N^{1 / 2}$ falls from approximately 6.32 to approximately 0.30 , which is easily verified. The appropriate critical values for the tests were also simulated with 10,000 replications but with $\gamma=0$ under the null. The size of the tests was set at $5 \%$ and one-tailed tests against the stationary alternative were considered. Tables 2 and 3 contain the simulation results. 
Table 2

Power of one-tailed test of unit root $(\gamma=0)$ against stationary alternative $\gamma=-0.2$, cases 1 (a) and $1(\mathrm{~b})$

\begin{tabular}{lccccccc}
\hline & & \multicolumn{7}{c}{$h$} \\
\cline { 3 - 8 } Statistic & $N$ & 2 & 1 & $\frac{1}{2}$ & $\frac{1}{4}$ & $\frac{1}{12}$ & $\frac{1}{52}$ \\
\hline Case 1(a): $y(0)=0$ & & & & & & \\
$T(\widehat{\alpha}-1)$ & 16 & 0.164 & 0.175 & 0.184 & 0.177 & 0.188 & 0.194 \\
& 32 & 0.373 & 0.419 & 0.420 & 0.458 & 0.427 & 0.450 \\
& 64 & 0.843 & 0.871 & 0.910 & 0.897 & 0.914 & 0.901 \\
& 128 & 0.999 & 1.000 & 1.000 & 1.000 & 1.000 & 1.000 \\
$T(\widetilde{\alpha}-1)$ & 16 & 0.128 & 0.159 & 0.178 & 0.170 & 0.183 & 0.194 \\
& 32 & 0.308 & 0.380 & 0.387 & 0.438 & 0.417 & 0.438 \\
& 64 & 0.733 & 0.809 & 0.886 & 0.883 & 0.908 & 0.902 \\
& 128 & 0.991 & 0.999 & 1.000 & 1.000 & 1.000 & 1.000
\end{tabular}

Case 1(b): $y(0)=0.1$

$$
\begin{array}{rrrrrrrr}
T^{2}(\widehat{\alpha}-1) & 16 & 0.158 & 0.175 & 0.184 & 0.188 & 0.346 & 0.952 \\
& 32 & 0.375 & 0.419 & 0.447 & 0.464 & 0.642 & 1.000 \\
& 64 & 0.841 & 0.883 & 0.902 & 0.930 & 0.989 & 1.000 \\
& 128 & 0.999 & 1.000 & 1.000 & 1.000 & 1.000 & 1.000 \\
T^{2}(\widetilde{\alpha}-1) & 16 & 0.129 & 0.145 & 0.156 & 0.134 & 0.235 & 0.707 \\
& 32 & 0.282 & 0.373 & 0.402 & 0.409 & 0.480 & 0.999 \\
& 64 & 0.718 & 0.818 & 0.869 & 0.904 & 0.955 & 1.000 \\
& 128 & 0.990 & 0.999 & 1.000 & 1.000 & 1.000 & 1.000
\end{array}
$$

Case 1(b): $y(0)=1$

$$
\begin{array}{rrrrrrrr}
T^{2}(\widehat{\alpha}-1) & 16 & 0.183 & 0.207 & 0.235 & 0.290 & 1.000 & 1.000 \\
& 32 & 0.414 & 0.556 & 0.773 & 0.998 & 1.000 & 1.000 \\
& 64 & 0.884 & 0.971 & 0.999 & 1.000 & 1.000 & 1.000 \\
& 128 & 1.000 & 1.000 & 1.000 & 1.000 & 1.000 & 1.000 \\
T^{2}(\widetilde{\alpha}-1) & 16 & 0.156 & 0.183 & 0.217 & 0.279 & 1.000 & 1.000 \\
& 32 & 0.264 & 0.314 & 0.315 & 0.860 & 1.000 & 1.000 \\
& 64 & 0.729 & 0.865 & 0.969 & 1.000 & 1.000 & 1.000 \\
& 128 & 0.993 & 1.000 & 1.000 & 1.000 & 1.000 & 1.000
\end{array}
$$


Table 3

Power of one-tailed test of unit root $(\gamma=0)$ against stationary alternative $\gamma=-0.2$, case $2(\mathrm{~b})(\mathrm{i})$

\begin{tabular}{lllllll}
\hline & \multicolumn{5}{c}{$N, h$} \\
\cline { 2 - 7 } Statistic & 10,2 & 12,1 & $16, \frac{1}{2}$ & $18, \frac{1}{4}$ & $36, \frac{1}{12}$ & $240, \frac{1}{52}$ \\
\hline$y(0)=0.1$ & & & & & & \\
$\frac{T^{2}}{N^{3 / 2}}(\widehat{\alpha}-1)$ & 0.102 & 0.125 & 0.181 & 0.213 & 0.723 & 1.000 \\
$\frac{T^{2}}{N^{3 / 2}}(\widetilde{\alpha}-1)$ & 0.082 & 0.113 & 0.154 & 0.167 & 0.464 & 1.000 \\
$y(0)=1$ & & & & & & \\
$\frac{T^{2}}{N^{3 / 2}}(\widehat{\alpha}-1)$ & 0.128 & 0.134 & 0.260 & 0.603 & 1.000 & 1.000 \\
$\frac{T^{2}}{N^{3 / 2}}(\widetilde{\alpha}-1)$ & 0.127 & 0.130 & 0.174 & 0.308 & 1.000 & 1.000 \\
$y(0)=10$ & & & & & & \\
$\frac{T^{2}}{N^{3 / 2}}(\widehat{\alpha}-1)$ & 0.617 & 1.000 & 1.000 & 1.000 & 1.000 & 1.000 \\
$\frac{T^{2}}{N^{3 / 2}}(\widetilde{\alpha}-1)$ & 0.019 & 1.000 & 1.000 & 1.000 & 1.000 & 1.000 \\
\hline
\end{tabular}

The first panel of Table 2 contains the results pertinent to case 1(a), in which span $N$ is fixed and frequency $h$ tends to zero. The entries should therefore be read along each row. According to Theorem 4 , the statistics $T(\widehat{\alpha}-1)$ and $T(\widetilde{\alpha}-1)$ are inconsistent, and this manifests itself in Table 2 by the simulated power not approaching unity as $h$ gets smaller. The high power when $N=128$ is a reflection of the span being sufficiently large for the test to have good power. The reason for this can be seen from Theorem 3, case 1(a), which shows that, under the alternative $\gamma \neq 0$,

$$
T_{n}\left(\widehat{\alpha}_{n}-1\right) \Rightarrow \frac{\Omega\left(J, W, \frac{1}{6}\right)}{\Psi\left(J^{2}\right)}+\gamma N, \quad T_{n}\left(\widetilde{\alpha}_{n}-1\right) \Rightarrow \frac{\Omega(J, W, 0)}{\Psi\left(J^{2}\right)}+\gamma N
$$

When $N$ is large, the term $\gamma N$ dominates this distribution, and with $\gamma<0$ the simulated values of $T_{n}\left(\widehat{\alpha}_{n}-1\right)$ and $T_{n}\left(\widetilde{\alpha}_{n}-1\right)$ take on large negative values which are significant. Note that, when $\gamma=-0.2$ and $N=128$, the product $\gamma N=-25.6$, while the largest simulated critical values (under the null) are -5.1 (OLS) and -11.2 (IV).

The second panel of Table 2 relates to case 1(b) which again holds $N$ fixed while $h$ 
tends to zero but in which $y(0) \neq 0$. In this case the statistics $T^{2}(\widehat{\alpha}-1)$ and $T^{2}(\widetilde{\alpha}-1)$ are consistent, according to Theorem 4, and indeed, even with a small value of $y(0)=0.1$, it is seen that the power tends to unity as the entries are read along each row. For larger values of $y(0)$ than those contained in Table 2, for example $y(0)=10$ or 100 , the power is unity for all reported entries except for that corresponding to $N=16, h=2$. Virtually identical results are obtained with negative values of $y(0)$, any differences arising due to sampling variability. That this is so is an implication of Theorem 2 which shows that the limiting distributions of these statistics are normal under the null, with the variance proportional to $y(0)^{-2}$.

In Table 3 the results relate to case 2(b)(i) in which span $N$ tends to infinity, frequency $h$ tends to zero while the product $h N^{1 / 2}$ also tends to zero. Under the null hypothesis the limiting distribution of the statistics is once again normal with variance proportional to $y(0)^{-2}$, and the results with the corresponding negative values of $y(0)$ are virtually identical to those reported for positive values of $y(0)$. The consistency of the statistics is evident from the entries in the Table. It is interesting to note, though, that the finite sample power is uniformly higher for the OLS-based test statistics than it is for the IV-based statistics, in both Tables 2 and 3 .

It is worthwhile, at this point, to reflect briefly on the results of other simulation studies of the power of unit root tests with flow data and varying sampling frequency, in light of the theoretical results obtained in this paper. Choi (1992) provides simulation results based on a fixed span of 100 periods and two frequencies, 1 and $1 / 4$, corresponding to annual and quarterly data respectively. With the initial value set equal to zero, his experimental design therefore corresponds to case 1(a) here. His main findings are that power is higher with the quarterly data than with the annual data, and that the Phillips-Perron tests are more powerful than the augmented Dickey-Fuller tests. The fact that power is found to be higher with the quarterly data is also seen in Table 2 , but the fact that we have shown such tests to be inconsistent is an important additional insight into these simulation findings. Ng (1995) conducts a more comprehensive investigation which also considers the IV-based statistic $T(\widetilde{\alpha}-1)$. Her simulations allow span and number of observations to vary between 25 and 1000 , hence yielding values of frequency between 40 and $1 / 40$. The value of $y(0)$ is taken to be zero and hence Ng's findings cover cases 1(a), 2(a) and 3(a). The fixed alternative is taken to be $\gamma=-0.2$, as here. Among Ng's findings are that increasing sample size while keeping span fixed increases power but at a diminishing rate. This is presumably a manifestation of test inconsistency shown in Theorem 4 for case 1(a). It is also possible to pick out from Ng's 
Table 1 of results the consistency of the tests in cases $2(\mathrm{a})$ and $3(\mathrm{a})$. In the former case this is achieved by reading down diagonally but jumping columns, so that span increases and frequency falls simultaneously, and in the latter case simply by reading down diagonally, so that frequency is fixed while span increases. All these simulation results are predicted by the theory derived in this paper.

\section{Concluding comments}

This paper has analysed the consistency properties of two tests for a unit root in flow data when the sampling frequency is allowed to vary. The limiting distributions of both the OLS-based statistic and the IV-based statistic are derived under both the null and alternative hypotheses, which enables the consistency properties of the tests to be established. Contrary to the situation with a stock variable, it is found that it is possible to consistently test for a unit root in a flow variable even when the span of the data is fixed, and, furthermore, that increasing the span is not in itself sufficient for consistent testing. The appropriate test statistic when span is fixed involves normalisation by the square of the sample size, implying a very fast convergence of the autoregressive coefficient estimator to unity under the null hypothesis. Now that time series data are becoming available at higher frequencies, the results contained in this paper provide some theoretical guidance to practitioners who wish to test for unit roots in flow variables. 


\section{Appendix}

Proof of Theorem 1. (a) The solution to (1) is given by

$$
y(t h)=e^{\gamma t h} y(0)+\sigma \int_{0}^{t h} e^{\gamma(t h-s)} d w(s),
$$

which yields the difference equation

$$
y(t h)=e^{\gamma h} y(t h-h)+\sigma \int_{t h-h}^{t h} e^{\gamma(t h-s)} d w(s) .
$$

Integrating (A2) once more over the interval $[t h-h, t h]$ yields an equation for flows with a disturbance term in the form of a double integral. This double integral representation can be avoided by following the approach of Bergstrom (1984) whose Theorem 8 derives the same equation for the case $h=1$. Integrating (1) over $[t h-h, t h]$ yields

$$
y(t h)-y(t h-h)=\gamma \int_{t h-h}^{t h} y(s) d s+\sigma \int_{t h-h}^{t h} d w(s)=\gamma y_{t h}+\sigma \int_{0}^{h} d w(t h-s),
$$

which can be solved for $y_{t h}$ to give

$$
y_{t h}=\gamma^{-1}[y(t h)-y(t h-h)]-\sigma \gamma^{-1} \int_{0}^{h} d w(t h-s) .
$$

From (A2),

$$
\begin{aligned}
y(t h)-y(t h-h)= & e^{\gamma h}[y(t h-h)-y(t h-2 h)] \\
& +\sigma\left[\int_{0}^{h} e^{\gamma r} d w(t h-s)-\int_{0}^{h} e^{\gamma r} d w(t h-h-r)\right] \\
= & e^{\gamma h}\left[\gamma \int_{0}^{h} y(t h-h-s) d s+\sigma \int_{0}^{h} d w(t h-h-s)\right] \\
& +\sigma\left[\int_{0}^{h} e^{\gamma s} d w(t h-s)-\int_{0}^{h} e^{\gamma s} d w(t h-h-s)\right] .
\end{aligned}
$$

Substituting (A4) into (A3) yields

$$
\begin{aligned}
y_{t h}= & \frac{e^{\gamma h}}{\gamma}\left[\gamma y_{t h-h}+\sigma \int_{0}^{h} d w(t h-h-s)\right] \\
& +\frac{\sigma}{\gamma}\left[\int_{0}^{h} e^{\gamma s} d w(t h-s)-\int_{0}^{h} e^{\gamma s} d w(t h-h-s)\right]-\frac{\sigma}{\gamma} \int_{0}^{h} d w(t h-s),
\end{aligned}
$$

which yields (3) upon rearranging the terms. The autocovariance properties of $u_{t h}$ then follow from its definition, with the orders of magnitude derived from expanding $e^{\gamma h}$ as the infinite series $\sum_{j=0}^{\infty}(\gamma h)^{j} / j$ ! and rearranging terms.

(b) Setting $\gamma=0$ and integrating (1) twice over $[t h-h, t h]$ yields (5) with $u_{t h}$ defined in 
terms of a double integral. Alternatively, $u_{t h}$ can be obtained by taking the limit of (4) as $\gamma \downarrow 0$, which yields (6) since

$$
\lim _{\gamma \downarrow 0} \frac{e^{\gamma s}-1}{\gamma}=s \text { and } \lim _{\gamma \downarrow 0} \frac{e^{\gamma h}-e^{\gamma s}}{\gamma}=h-s .
$$

The autocovariances then follow from (6).

Proof of Lemma 1. The objective is to express the sample moments in terms of the random function $X_{n}(r)$. This is achieved by noting that

$$
\begin{aligned}
& S_{n t-1}=S_{n t-1} T_{n} \int_{(t-1) / T_{n}}^{t / T_{n}} d r=T_{n} \int_{(t-1) / T_{n}}^{t / T_{n}} S_{n\left[T_{n} r\right]} d r=T_{n}^{3 / 2} h_{n}^{3 / 2} \int_{(t-1) / T_{n}}^{t / T_{n}} X_{n}(r) d r, \\
& S_{n t-1}^{2}=S_{n t-1}^{2} T_{n} \int_{(t-1) / T_{n}}^{t / T_{n}} d r=T_{n} \int_{(t-1) / T_{n}}^{t / T_{n}} S_{n\left[T_{n} r\right]}^{2} d r=T_{n}^{2} h_{n}^{3} \int_{(t-1) / T_{n}}^{t / T_{n}} X_{n}(r)^{2} d r .
\end{aligned}
$$

This enables the sample moments to be written

$$
\begin{aligned}
\sum_{t=1}^{T_{n}} y_{n t-1} u_{n t} & =\sum_{t=1}^{T_{n}}\left(S_{n t-1}+y_{n 0}\right) u_{n t} \\
& =\frac{1}{2} \sum_{t=1}^{T_{n}}\left(S_{n t}^{2}-S_{n t-1}^{2}-u_{n t}^{2}\right)+y(0) \sum_{t=1}^{T_{n}} u_{n t} \\
& =\frac{1}{2}\left(S_{n T_{n}}^{2}-\sum_{t=1}^{T_{n}} u_{n t}^{2}\right)+y(0) S_{n T_{n}} \\
& =\frac{T_{n} h_{n}^{3}}{2} X_{n}(1)^{2}-\frac{1}{2} \sum_{t=1}^{T_{n}} u_{n t}^{2}+T_{n}^{1 / 2} h_{n}^{3 / 2} y(0) X_{n}(1) \\
& =\frac{N_{n} h_{n}^{2}}{2} X_{n}(1)^{2}-\frac{h_{n}^{2} N_{n}}{2} \frac{1}{T_{n}} \sum_{t=1}^{T_{n}}\left(u_{n t}^{h}\right)^{2}+N_{n}^{1 / 2} h_{n} y(0) X_{n}(1),
\end{aligned}
$$

and in a similar fashion

$$
\begin{aligned}
\sum_{t=1}^{T_{n}} y_{n t-1}^{2} & =\sum_{t=1}^{T_{n}}\left(S_{n t-1}+y_{n 0}\right)^{2} \\
& =\sum_{t=1}^{T_{n}} S_{n t-1}^{2}+2 y(0) \sum_{t=1}^{T_{n}} S_{n t-1}+T_{n} y(0)^{2} \\
& =T_{n}^{2} h_{n}^{3} \int_{0}^{1} X_{n}(r)^{2} d r+2 y(0) T_{n}^{3 / 2} h_{n}^{3 / 2} \int_{0}^{1} X_{n}(r) d r+T_{n} y(0)^{2} .
\end{aligned}
$$

The sample moments for the IV estimator can be obtained using (A5) and (A6) above allied with the representations (12) and (13) in the text, which only requires, in addition to the above, the limit of the appropriately normalised moment $\sum_{t=1}^{T_{n}} u_{n t} u_{n t-1}$. Taking each case 
in turn (all limits are taken as $n \uparrow \infty$ ):

Case 1(a): From (A5) and (A6) with $y(0)=0$,

$$
\begin{aligned}
& \frac{1}{h_{n}^{2}} \sum_{t=1}^{T_{n}} y_{n t-1} u_{n t}=\frac{N}{2} X_{n}(1)^{2}-\frac{N}{2} \frac{1}{T_{n}} \sum_{t=1}^{T_{n}}\left(u_{n t}^{h}\right)^{2} \Rightarrow \frac{\sigma^{2} N}{2} W(1)^{2}-\frac{N}{2} \frac{2}{3} \sigma^{2}, \\
& \frac{1}{h_{n}} \sum_{t=1}^{T_{n}} y_{n t-1}^{2}=N^{2} \int_{0}^{1} X_{n}(r)^{2} d r \Rightarrow \sigma^{2} N^{2} \int_{0}^{1} W(r)^{2} d r, \\
& \frac{1}{h_{n}^{2}} \sum_{t=1}^{T_{n}} u_{n t} u_{n t-1}=\frac{N}{T_{n}} \sum_{t=1}^{T_{n}} u_{n t}^{h} u_{n t-1}^{h} \stackrel{p}{\rightarrow} \frac{N \sigma^{2}}{6} .
\end{aligned}
$$

Case 1(b): From (A5) and (A6),

$$
\begin{aligned}
& \frac{1}{h_{n}} \sum_{t=1}^{T_{n}} y_{n t-1} u_{n t}=\frac{N h_{n}}{2} X_{n}(1)^{2}-\frac{N^{2}}{2 T_{n}^{2}} \sum_{t=1}^{T_{n}}\left(u_{n t}^{h}\right)^{2}+N^{1 / 2} y(0) X_{n}(1) \Rightarrow \sigma N^{1 / 2} y(0) W(1), \\
& h_{n} \sum_{t=1}^{T_{n}} y_{n t-1}^{2}=h_{n}^{2} N^{2} \int_{0}^{1} X_{n}(r)^{2} d r+2 h_{n} N^{3 / 2} y(0) \int_{0}^{1} X_{n}(r) d r+N y(0)^{2} \stackrel{p}{\rightarrow} N y(0)^{2}, \\
& \frac{1}{h_{n}} \sum_{t=1}^{T_{n}} u_{n t} u_{n t-1}=\frac{h_{n} N}{T_{n}} \sum_{t=1}^{T_{n}} u_{n t}^{h} u_{n t-1}^{h} \stackrel{p}{\rightarrow} 0 .
\end{aligned}
$$

Case 2(a): From (A5) and (A6),

$$
\begin{aligned}
& \frac{1}{h_{n}^{2} N_{n}} \sum_{t=1}^{T_{n}} y_{n t-1} u_{n t}=\frac{1}{2} X_{n}(1)^{2}-\frac{1}{2} \frac{1}{T_{n}} \sum_{t=1}^{T_{n}}\left(u_{n t}^{h}\right)^{2} \Rightarrow \frac{\sigma^{2}}{2}\left[W(1)^{2}-\frac{2}{3}\right]=\Phi(W, 2 / 3), \\
& \frac{1}{h_{n} N_{n}^{2}} \sum_{t=1}^{T_{n}} y_{n t-1}^{2}=\int_{0}^{1} X_{n}(r)^{2} d r \Rightarrow \sigma^{2} \int_{0}^{1} W(r)^{2} d r=\Psi\left(W^{2}\right), \\
& \frac{1}{h_{n}^{2} N_{n}} \sum_{t=1}^{T_{n}} u_{n t} u_{n t-1}=\frac{1}{T_{n}} \sum_{t=1}^{T_{n}} u_{n t}^{h} u_{n t-1}^{h} \stackrel{p}{\rightarrow} \frac{\sigma^{2}}{6} .
\end{aligned}
$$

Case 2(b)(i): From (A5) and (A6),

$$
\begin{aligned}
& \frac{1}{h_{n} N_{n}^{1 / 2}} \sum_{t=1}^{T_{n}} y_{n t-1} u_{n t}=\frac{h_{n} N_{n}^{1 / 2}}{2} X_{n}(1)^{2}-\frac{h_{n} N_{n}^{1 / 2}}{2 T_{n}} \sum_{t=1}^{T_{n}}\left(u_{n t}^{h}\right)^{2}+y(0) X_{n}(1) \Rightarrow \sigma y(0) W(1), \\
& \frac{h_{n}}{N_{n}} \sum_{t=1}^{T_{n}} y_{n t-1}^{2}=h_{n}^{2} N_{n} \int_{0}^{1} X_{n}(r)^{2} d r+2 h_{n} N_{n}^{1 / 2} \int_{0}^{1} X_{n}(r) d r+y(0)^{2} \stackrel{p}{\rightarrow} y(0)^{2}, \\
& \frac{1}{h_{n} N_{n}^{1 / 2}} \sum_{t=1}^{T_{n}} u_{n t} u_{n t-1}=\frac{h_{n} N_{n}^{1 / 2}}{T_{n}} \sum_{t=1}^{T_{n}} u_{n t}^{h} u_{n t-1}^{h} \stackrel{p}{\rightarrow} 0 .
\end{aligned}
$$


Case 2(b)(ii): From (A5) and (A6),

$$
\begin{aligned}
& \sum_{t=1}^{T_{n}} y_{n t-1} u_{n t} \Rightarrow \frac{k^{2} \sigma^{2}}{2}\left[W(1)^{2}-\frac{2}{3}\right]+k \sigma y(0) W(1)=k^{2} \Phi(W, 2 / 3)+k \sigma y(0) W(1), \\
& \frac{h_{n}}{N_{n}} \sum_{t=1}^{T_{n}} y_{n t-1}^{2} \Rightarrow k^{2} \sigma^{2} \int_{0}^{1} W(r)^{2} d r+2 k y(0) \sigma \int_{0}^{1} W(r) d r+y(0)^{2}, \\
& \sum_{t=1}^{T_{n}} u_{n t} u_{n t-1}=\frac{h_{n}^{2} N_{n}}{T_{n}} \sum_{t=1}^{T_{n}} u_{n t}^{h} u_{n t-1}^{h} \stackrel{p}{\rightarrow} \frac{k^{2} \sigma^{2}}{6} .
\end{aligned}
$$

Case 2(b)(iii): From (A5) and (A6),

$$
\begin{aligned}
& \frac{1}{h_{n}^{2} N_{n}} \sum_{t=1}^{T_{n}} y_{n t-1} u_{n t}=\frac{1}{2} X_{n}(1)^{2}-\frac{1}{2 T_{n}} \sum_{t=1}^{T_{n}}\left(u_{n t}^{h}\right)^{2}+\frac{1}{h_{n} N_{n}^{1 / 2}} y(0) X_{n}(1) \Rightarrow \frac{\sigma^{2}}{2}\left[W(1)^{2}-\frac{2}{3}\right], \\
& \frac{1}{h_{n} N_{n}^{2}} \sum_{t=1}^{T_{n}} y_{n t-1}^{2}=\int_{0}^{1} X_{n}(r)^{2} d r+\frac{2}{h_{n} N_{n}^{1 / 2}} y(0) \int_{0}^{1} X_{n}(r) d r+\frac{1}{h_{n}^{2} N_{n}} y(0)^{2} \Rightarrow \sigma^{2} \int_{0}^{1} W(r)^{2} d r \\
& \frac{1}{h_{n}^{2} N_{n}} \sum_{t=1}^{T_{n}} u_{n t} u_{n t-1}=\frac{1}{T_{n}} \sum_{t=1}^{T_{n}} u_{n t}^{h} u_{n t-1}^{h} \stackrel{p}{\rightarrow} \frac{\sigma^{2}}{6} .
\end{aligned}
$$

Case 3: From (A5) and (A6),

$$
\begin{aligned}
& \frac{1}{T_{n}} \sum_{t=1}^{T_{n}} y_{n t-1} u_{n t}=\frac{h^{3}}{2} X_{n}(1)^{2}-\frac{1}{2 T_{n}} \sum_{t=1}^{T_{n}}\left(u_{n t}^{h}\right)^{2}+\frac{h^{3 / 2}}{T_{n}^{1 / 2}} y(0) X_{n}(1) \Rightarrow \frac{h^{3} \sigma^{2}}{2}\left[W(1)^{2}-\frac{2}{3}\right], \\
& \frac{1}{T_{n}^{2}} \sum_{t=1}^{T_{n}} y_{n t-1}^{2}=h^{3} \int_{0}^{1} X_{n}(r)^{2} d r+2 y(0) \frac{h^{3 / 2}}{T_{n}^{1 / 2}} \int_{0}^{1} X_{n}(r) d r+\frac{1}{T_{n}} y(0)^{2} \Rightarrow h^{3} \sigma^{2} \int_{0}^{1} W(r)^{2} d r, \\
& \frac{1}{T_{n}} \sum_{t=1}^{T_{n}} u_{n t} u_{n t-1}=\frac{h^{3}}{T_{n}} \sum_{t=1}^{T_{n}} u_{n t}^{h} u_{n t-1}^{h} \stackrel{p}{\rightarrow} \frac{h^{3} \sigma^{2}}{6} .
\end{aligned}
$$

This completes the proof.

Proof of Theorem 2. The limiting distributions are obtained from the convergence results for the sample moments in Lemma 1. In the case of the OLS estimator, if the convergence of $\tau_{1}(n) \sum_{t=1}^{T_{n}} y_{n t-1} u_{n t}$ and $\tau_{2}(n) \sum_{t=1}^{T_{n}} y_{n t-1}^{2}$ have been established, then $\left[\tau_{1}(n) / \tau_{2}(n)\right]\left(\widehat{\alpha}_{n}-1\right)$ has the limiting distribution stated in the Theorem. A similar argument holds for the IV 
estimator. Taking each case in turn:

Case 1(a): From Lemma 1,

$$
\frac{1}{h_{n}}\left(\widehat{\alpha}_{n}-1\right) \Rightarrow \frac{\frac{1}{2}\left[W(1)^{2}-\frac{2}{3}\right]}{N \int_{0}^{1} W(r)^{2} d r}, \frac{1}{h_{n}}\left(\widetilde{\alpha}_{n}-1\right) \Rightarrow \frac{\frac{1}{2}\left[W(1)^{2}-1\right]}{N \int_{0}^{1} W(r)^{2} d r} .
$$

Multiplying by $N$ gives $T_{n}\left(\widehat{\alpha}_{n}-1\right) \Rightarrow Z(W, 2 / 3)$ and $T_{n}\left(\widetilde{\alpha}_{n}-1\right) \Rightarrow Z(W, 1)$ as required.

Case 1(b): From Lemma 1,

$$
\frac{1}{h_{n}^{2}}\left(\widehat{\alpha}_{n}-1\right) \Rightarrow \frac{\sigma W(1)}{N^{1 / 2} y(0)}, \quad \frac{1}{h_{n}^{2}}\left(\widetilde{\alpha}_{n}-1\right) \Rightarrow \frac{\sigma W(1)}{N^{1 / 2} y(0)}
$$

Multiplying by $N^{2}$ gives

$$
T_{n}^{2}\left(\widehat{\alpha}_{n}-1\right) \Rightarrow \frac{N^{3 / 2} \sigma W(1)}{y(0)} \text { and } T_{n}^{2}\left(\widetilde{\alpha}_{n}-1\right) \Rightarrow \frac{N^{3 / 2} \sigma W(1)}{y(0)}
$$

as required.

Case 2(a): Noting that $\left(h_{n}^{-2} N_{n}^{-1}\right) /\left(h_{n}^{-1} N_{n}^{-2}\right)=N_{n} / h_{n}=T_{n}$ yields the results in a straightforward fashion.

Case 2(b)(i): Noting that $\left(h_{n}^{-1} N_{n}^{-1 / 2}\right) /\left(h_{n} N_{n}^{-1}\right)=T_{n}^{2} / N_{n}^{3 / 2}$ yields

$$
\frac{T_{n}^{2}}{N_{n}^{3 / 2}}\left(\widehat{\alpha}_{n}-1\right) \Rightarrow \frac{\sigma W(1)}{y(0)}, \quad \frac{T_{n}^{2}}{N_{n}^{3 / 2}}\left(\widetilde{\alpha}_{n}-1\right) \Rightarrow \frac{\sigma W(1)}{y(0)},
$$

as required.

Case 2(b)(ii): Since $1 /\left(h_{n} N_{n}^{-1}\right)=T_{n}$ the distributions for $T_{n}\left(\widehat{\alpha}_{n}-1\right)$ and $T_{n}\left(\widetilde{\alpha}_{n}-1\right)$ follow immediately from Lemma 1.

Case 2(b)(iii): This follows in the same way as case 2(a).

Case 3: The normalisation here is familiar and the $h^{3}$ factors cancel out in the numerator and denominator of both estimators, giving the stated distributions.

Proof of Lemma 2. Once again the objective is to express the sample moments in terms of the function $X_{n}(r)$. From (7) it follows that

$$
y_{n t}=\sum_{j=1}^{t} e^{(t-j) \gamma h_{n}} u_{n j}+e^{t \gamma h_{n}} y_{n 0}
$$

which yields

$$
y_{n T_{n}}=h_{n}^{3 / 2} \sum_{j=1}^{t} e^{\left(T_{n}-j\right) \gamma N_{n} / T_{n}} u_{n j}^{h}+e^{T_{n} \gamma h_{n}} y(0)
$$




$$
\begin{aligned}
& =h_{n}^{3 / 2} \sum_{j=1}^{t} e^{\left(T_{n}-j\right) \gamma N_{n} / T_{n}} \int_{(j-1) / T_{n}}^{j / T_{n}} T_{n}^{1 / 2} d X_{n}(s)+e^{\gamma N_{n}} y(0) \\
& =h_{n} N_{n}^{1 / 2} \int_{0}^{1} e^{(1-s) \gamma N_{n}} d X_{n}(s)+e^{\gamma N_{n}} y(0) .
\end{aligned}
$$

Furthermore

$$
\begin{aligned}
\sum_{t=1}^{T_{n}} y_{n t}^{2}= & \sum_{t=1}^{T_{n}}\left(\sum_{j=1}^{t} e^{(t-j) \gamma h_{n}} u_{n j}+e^{t \gamma h_{n}} y_{n 0}\right)^{2} \\
= & \sum_{t=1}^{T_{n}}\left(\sum_{j=1}^{t} e^{(t-j) \gamma N_{n} / T_{n}} u_{n j}\right)^{2}+2 y(0) \sum_{t=1}^{T_{n}} e^{t \gamma N_{n} / T_{n}} \sum_{j=1}^{t} e^{(t-j) \gamma N_{n} / T_{n}} u_{n j} \\
& +y(0)^{2} \sum_{t=1}^{T_{n}} e^{2 t \gamma N_{n} / T_{n}}=A_{1 n}+A_{2 n}+A_{3 n} .
\end{aligned}
$$

Considering each term in turn:

$$
\begin{aligned}
A_{1 n} & =h_{n}^{3} \sum_{t=1}^{T_{n}}\left(\sum_{j=1}^{t} e^{(t-j) \gamma N_{n} / T_{n}} u_{n j}^{h}\right)^{2} \\
& =h_{n}^{3} \sum_{t=1}^{T_{n}}\left(\sum_{j=1}^{t} e^{(t-j) \gamma N_{n} / T_{n}} \int_{(j-1) / T_{n}}^{j / T_{n}} T_{n}^{1 / 2} d X_{n}(s)\right)^{2} \\
& =T_{n} h_{n}^{3} \sum_{t=1}^{T_{n}} T_{n} \int_{(t-1) / T_{n}}^{t / T_{n}}\left(\sum_{j=1}^{t} e^{(t-j) \gamma N_{n} / T_{n}} \int_{(j-1) / T_{n}}^{j / T_{n}} d X_{n}(s)\right)^{2} d r \\
& =h_{n} N_{n}^{2} \int_{0}^{1}\left(\int_{0}^{r} e^{(r-s) \gamma N_{n}} d X_{n}(s)\right)^{2} d r ; \\
A_{2 n} & =2 y(0) h_{n}^{3 / 2} \sum_{t=1}^{T_{n}} e^{t \gamma N_{n} / T_{n}} \sum_{j=1}^{t} e^{(t-j) \gamma N_{n} / T_{n}} u_{n j}^{h} \\
& =2 y(0) h_{n}^{3 / 2} \sum_{t=1}^{T_{n}} e^{t \gamma N_{n} / T_{n}} \sum_{j=1}^{t} e^{(t-j) \gamma N_{n} / T_{n}} \int_{(j-1) / T_{n}}^{j / T_{n}} T_{n}^{1 / 2} d X_{n}(s) \\
& =2 y(0) T_{n}^{1 / 2} h_{n}^{3 / 2} \sum_{t=1}^{T_{n}} e^{t \gamma N_{n} / T_{n}} T_{n} \int_{(t-1) / T_{n}}^{t / T_{n}}\left(\sum_{j=1}^{t} e^{(t-j) \gamma N_{n} / T_{n}} \int_{(j-1) / T_{n}}^{j / T_{n}} d X_{n}(s)\right) d r \\
& =2 y(0) N_{n}^{3 / 2} \int_{0}^{1} e^{r \gamma N_{n}}\left(\int_{0}^{r} e^{(r-s) \gamma N_{n}} d X_{n}(s)\right) d r .
\end{aligned}
$$

Combining the expressions for $A_{1 n}, A_{2 n}$ and $A_{3 n}$ yields

$$
\begin{aligned}
\sum_{t=1}^{T_{n}} y_{n t}^{2}= & h_{n} N_{n}^{2} \int_{0}^{1}\left(\int_{0}^{r} e^{(r-s) \gamma N_{n}} d X_{n}(s)\right)^{2} d r \\
& +2 y(0) N_{n}^{3 / 2} \int_{0}^{1} e^{r \gamma N_{n}}\left(\int_{0}^{r} e^{(r-s) \gamma N_{n}} d X_{n}(s)\right) d r+y(0)^{2} \sum_{t=1}^{T_{n}} e^{2 t \gamma N_{n} / T_{n}} .
\end{aligned}
$$


An expression for $\sum_{t=1}^{T_{n}} y_{n t-1} u_{n t}$ can be obtained by squaring both sides of (A7) to give

$$
y_{n t}^{2}=e^{2 \gamma h_{n}} y_{n t-1}^{2}+2 e^{\gamma h_{n}} y_{n t-1} u_{n t}+u_{n t}^{2} .
$$

Subtracting $y_{n t-1}^{2}$ from both sides and summing from $t=1$ to $t=T_{n}$ gives

$$
y_{n T_{n}}^{2}-y_{n 0}^{2}=\left(e^{2 \gamma h_{n}}-1\right) \sum_{t=1}^{T_{n}} y_{n t-1}^{2}+2 e^{\gamma h_{n}} \sum_{t=1}^{T_{n}} y_{n t-1} u_{n t}+\sum_{t=1}^{T_{n}} u_{n t}^{2},
$$

which can be solved to give

$$
\sum_{t=1}^{T_{n}} y_{n t-1} u_{n t}=\frac{e^{-\gamma h_{n}}}{2}\left(y_{n T_{n}}^{2}-y_{n 0}^{2}-\left(e^{2 \gamma h_{n}}-1\right) \sum_{t=1}^{T_{n}} y_{n t-1}^{2}-h_{n}^{3} \sum_{t=1}^{T_{n}}\left(u_{n t}^{h}\right)^{2}\right)
$$

These expressions are required for the OLS estimator, and combined with (14) and (15) in the text, also provide the results for the IV estimator once the limiting properties of $\sum_{t=1}^{T_{n}} u_{n t} u_{n t-1}$ are established. Taking each case in turn:

Case (1a): From (A8), with $y(0)=0$ and $N_{n}=N, h_{n}^{-1} y_{n T_{n}} \Rightarrow N^{1 / 2} \sigma J(1)$. Furthermore,

$$
\frac{1}{h_{n}} \sum_{t=1}^{T_{n}} y_{n t}^{2} \Rightarrow N^{2} \sigma^{2} \int_{0}^{1} J(r)^{2} d r
$$

while

$$
\begin{aligned}
\frac{1}{h_{n}^{2}} \sum_{t=1}^{T_{n}} y_{n t-1} u_{n t} & =\frac{e^{-\gamma h_{n}}}{2}\left[\left(\frac{1}{h_{n}} y_{n T_{n}}\right)^{2}-\frac{1}{h_{n}}\left(e^{2 \gamma h_{n}}-1\right) \frac{1}{h_{n}} \sum_{t=1}^{T_{n}} y_{n t-1}^{2}-\frac{N}{T_{n}} \sum_{t=1}^{T_{n}}\left(u_{n t}^{h}\right)^{2}\right] \\
& \Rightarrow \frac{N \sigma^{2}}{2}\left(J(1)^{2}-2 \gamma N \int_{0}^{1} J(r)^{2} d r-\frac{2}{3}\right),
\end{aligned}
$$

which holds because, as $n \uparrow \infty$ and $h_{n} \downarrow 0, e^{-\gamma h_{n}} \rightarrow 1$ and $\left(e^{2 \gamma h_{n}}-1\right) / h_{n}=h_{n}^{-1}\left(2 \gamma h_{n}+\right.$ $\left.O\left(h_{n}^{2}\right)\right) \rightarrow 2 \gamma$. Now, from equation (8) of Phillips (1987b),

$$
J(1)^{2}=1+2 \gamma N \int_{0}^{1} J(r)^{2} d r+2 \int_{0}^{1} J(r) d W(r),
$$

from which it can be established that

$$
\frac{1}{2}\left(J(1)^{2}-2 \gamma N \int_{0}^{1} J(r)^{2} d r-\frac{2}{3}\right)=\int_{0}^{1} J(r) d W(r)+\frac{1}{6} .
$$

Finally,

$$
\frac{1}{h_{n}^{2}} \sum_{t=1}^{T_{n}} u_{n t} u_{n t-1}=\frac{N}{T_{n}} \sum_{t=1}^{T_{n}} u_{n t}^{h} u_{n t-1}^{h} \stackrel{p}{\rightarrow} \frac{N \sigma^{2}}{6} .
$$


Case 1(b): From (7), $y_{n T_{n}} \stackrel{p}{\rightarrow} e^{\gamma N} y(0)$. When $y(0) \neq 0$, it is necessary to consider all three components that constitute $\sum_{t=1}^{T_{n}} y_{n t}^{2}$. It was shown, in case $1(\mathrm{a})$, that $h_{n}^{-1} A_{1 n}$ converges to a well defined limit, and it is also holds that

$$
A_{2 n} \Rightarrow 2 y(0) N^{3 / 2} \int_{0}^{1} e^{\gamma N r} J(r) d r .
$$

Turning to $A_{3 n}$, it can be shown that

$$
\frac{2 \gamma N}{T_{n}} \sum_{t=1}^{T_{n}} e^{2 t \gamma N / T_{n}} \rightarrow e^{2 \gamma N}-1 \text { as } n \uparrow \infty,
$$

and so $T_{n}^{-1} A_{3 n} \rightarrow y(0)^{2}\left(e^{2 \gamma N}-1\right) /(2 \gamma N)$. This can be written alternatively as $h_{n} A_{3 n} \rightarrow$ $y(0)^{2}\left(e^{2 \gamma N}-1\right) /(2 \gamma)$, which establishes the result in Lemma 2 because $h_{n} A_{1 n}$ and $h_{n} A_{2 n}$ both converge to zero. Finally,

$$
\begin{aligned}
\sum_{t=1}^{T_{n}} y_{n t-1} u_{n t} & =\frac{e^{-\gamma h_{n}}}{2}\left[\left(y_{n T_{n}}\right)^{2}-y(0)^{2}-\frac{1}{h_{n}}\left(e^{2 \gamma h_{n}}-1\right) h_{n} \sum_{t=1}^{T_{n}} y_{n t-1}^{2}-\frac{h_{n}^{2} N}{T_{n}} \sum_{t=1}^{T_{n}}\left(u_{n t}^{h}\right)^{2}\right] \\
& \stackrel{p}{\rightarrow} \frac{1}{2}\left(e^{2 \gamma N} y(0)^{2}-y(0)^{2}-2 \gamma y(0)^{2} \frac{\left(e^{2 \gamma N}-1\right)}{2 \gamma}\right)=0,
\end{aligned}
$$

while

$$
\sum_{t=1}^{T_{n}} u_{n t} u_{n t-1}=\frac{h_{n}^{2} N}{T_{n}} \sum_{t=1}^{T_{n}} u_{n t}^{h} u_{n t-1}^{h} \stackrel{p}{\rightarrow} 0 .
$$

Case 2(a): From case 1(a) it is known that, as $h_{n} \downarrow 0$ with $N$ fixed,

$$
\frac{1}{h_{n}^{2}} \sum_{t=1}^{T_{n}} y_{n t-1} u_{n t} \Rightarrow \frac{N \sigma^{2}}{2}\left(\int_{0}^{1} J(r) d W(r)+\frac{1}{6}\right), \quad \frac{1}{h_{n}} \sum_{t=1}^{T_{n}} y_{n t}^{2} \Rightarrow N^{2} \sigma^{2} \int_{0}^{1} J(r)^{2} d r .
$$

When $\gamma>0$ and defining $c=\gamma N_{n}$, Lemma 2 of Phillips (1987b) shows that, with $J(r)=$ $\int_{0}^{r} e^{(r-s) c} d W(s)$,

$$
\frac{2 c}{e^{c}} \int_{0}^{1} J(r) d W(r) \Rightarrow \xi \eta, \quad \frac{(2 c)^{2}}{e^{2 c}} \int_{0}^{1} J(r)^{2} d r \Rightarrow \eta^{2} \quad \text { as } c \uparrow \infty,
$$

where $\xi$ and $\eta$ are independent standard normal random variables, Furthermore,

$$
\frac{2 \gamma N_{n}}{e^{\gamma N_{n}} h_{n}^{2} N_{n}} \sum_{t=1}^{T_{n}} u_{n t} u_{n t-1}=\frac{2 \gamma N_{n}}{e^{\gamma N_{n}} T_{n}} \sum_{t=1}^{T_{n}} u_{n t}^{h} u_{n t-1}^{h} \stackrel{p}{\rightarrow} 0,
$$

and the results follow for the $\gamma>0$ case. When $\gamma<0$, the results once more utilise Lemma 2 of Phillips (1987b) which shows that

$$
(-2 c)^{1 / 2} \int_{0}^{1} J(r) d W(r) \Rightarrow N(0,1), \quad-2 c \int_{0}^{1} J(r)^{2} d r \stackrel{p}{\rightarrow} 1 \text { as } c \downarrow-\infty .
$$


Finally, although

$$
\frac{\left(-2 \gamma N_{n}\right)^{1 / 2}}{h_{n}^{2} N_{n}} \sum_{t=1}^{T_{n}} u_{n t} u_{n t-1}=\frac{\left(-2 \gamma N_{n}\right)^{1 / 2}}{T_{n}} \sum_{t=1}^{T_{n}} u_{n t}^{h} u_{n t-1}^{h} \stackrel{p}{\rightarrow} \infty,
$$

an alternative normalisation does yield a finite limit, namely

$$
\frac{1}{h_{n}^{2} N_{n}} \sum_{t=1}^{T_{n}} u_{n t} u_{n t-1}=\frac{1}{T_{n}} \sum_{t=1}^{T_{n}} u_{n t}^{h} u_{n t-1}^{h} \stackrel{p}{\rightarrow} \frac{\sigma^{2}}{6}
$$

which determines the normalisation for $\sum_{t=1}^{T_{n}} y_{n t-2} u_{n t}$ using (14).

Case 2(b): When $\gamma>0$ it follows from case 1(b) that

$$
\frac{1}{e^{2 \gamma N_{n}}} y_{n T_{n}}^{2} \stackrel{p}{\rightarrow} y(0)^{2}, \quad \frac{h_{n}}{e^{2 \gamma N_{n}}} \sum_{t=1}^{T_{n}} y_{n t-1}^{2} \stackrel{p}{\rightarrow} \frac{y(0)^{2}}{2 \gamma}
$$

Hence from (A9),

$$
\begin{aligned}
& \frac{1}{e^{2 \gamma N_{n}}} \sum_{t=1}^{T_{n}} y_{n t-1} u_{n t} \\
& \quad=\frac{e^{-\gamma h_{n}}}{2}\left(\frac{y_{n T_{n}}^{2}-y_{n 0}^{2}}{e^{2 \gamma N_{n}}}-\frac{\left(e^{2 \gamma h_{n}}-1\right)}{h_{n}} \frac{h_{n}}{e^{2 \gamma N_{n}}} \sum_{t=1}^{T_{n}} y_{n t-1}^{2}-\frac{h_{n}^{2} N_{n}}{e^{2 \gamma N_{n}} T_{n}} \sum_{t=1}^{T_{n}}\left(u_{n t}^{h}\right)^{2}\right) \\
& \stackrel{p}{\rightarrow} y(0)^{2}-2 \gamma \frac{y(0)^{2}}{2 \gamma}=0 .
\end{aligned}
$$

The results applicable to the IV estimator then follow because

$$
\frac{1}{e^{2 \gamma N_{n}}} \sum_{t=1}^{T_{n}} u_{n t} u_{n t-1}=\frac{h_{n}^{3}}{e^{2 \gamma N_{n}}} \sum_{t=1}^{T_{n}} u_{n t}^{h} u_{n t-1}^{h} \stackrel{p}{\rightarrow} 0
$$

Case 2(b)(i): When $\gamma<0$ the normalisation of the components in (A9) changes. It is now the case that $e^{\gamma N_{n}} \downarrow 0$ as $n \uparrow \infty$ so that

$$
y_{n T_{n}} \stackrel{p}{\rightarrow} 0, \quad \frac{1}{e^{\gamma N_{n}}} y_{n T_{n}} \stackrel{p}{\rightarrow} y(0), \quad h_{n} \sum_{t=1}^{T_{n}} y_{n t-1}^{2} \stackrel{p}{\rightarrow}-\frac{y(0)^{2}}{2 \gamma}
$$

the latter limit being positive as required since $\gamma<0$. Since, from (A9),

$$
\sum_{t=1}^{T_{n}} y_{n t-1} u_{n t}=\frac{e^{-\gamma h_{n}}}{2}\left(y_{n T_{n}}^{2}-y_{n 0}^{2}-\left(e^{2 \gamma h_{n}}-1\right) \sum_{t=1}^{T_{n}} y_{n t-1}^{2}-\frac{h_{n}^{2} N_{n}}{T_{n}} \sum_{t=1}^{T_{n}}\left(u_{n t}^{h}\right)^{2}\right)
$$

it follows that

$$
\sum_{t=1}^{T_{n}} y_{n t-1} u_{n t} \stackrel{p}{\rightarrow}-y(0)^{2}-2 \gamma\left(-\frac{y(0)^{2}}{2 \gamma}\right)=0
$$


The IV results follow because, when $h_{n} N_{n}^{1 / 2} \downarrow 0$,

$$
\sum_{t=1}^{T_{n}} u_{n t} u_{n t-1}=\frac{h_{n}^{2} N_{n}}{T_{n}} \sum_{t=1}^{T_{n}} u_{n t}^{h} u_{n t-1}^{h} \stackrel{p}{\rightarrow} 0 .
$$

Case 2(b)(ii): When $\gamma<0$ and $h_{n} N_{n}^{1 / 2} \rightarrow k$ as $n \uparrow \infty$, the arguments in 2(b)(i) yield

$$
\begin{aligned}
& \sum_{t=1}^{T_{n}} y_{n t-1} u_{n t} \stackrel{p}{\rightarrow}-y(0)^{2}-2 \gamma\left(-\frac{y(0)^{2}}{2 \gamma}\right)-\frac{2 k^{2} \sigma^{2}}{3}=-\frac{2 k^{2} \sigma^{2}}{3}, \\
& \sum_{t=1}^{T_{n}} u_{n t} u_{n t-1}=\frac{h_{n}^{2} N_{n}}{T_{n}} \sum_{t=1}^{T_{n}} u_{n t}^{h} u_{n t-1}^{h} \stackrel{p}{\rightarrow} \frac{k^{2} \sigma^{2}}{6} .
\end{aligned}
$$

Case 2(b)(iii): When $\gamma<0$ and $h_{n} N_{n}^{1 / 2} \uparrow \infty$ as $n \uparrow \infty$,

$$
\begin{aligned}
& \frac{1}{h_{n}^{2} N_{n}} \sum_{t=1}^{T_{n}} y_{n t-1} u_{n t} \\
& =\frac{e^{-\gamma h_{n}}}{2}\left(\frac{y_{n T_{n}}^{2}-y_{n 0}^{2}}{h_{n}^{2} N_{n}}-\frac{\left(e^{2 \gamma h_{n}}-1\right)}{h_{n}} \frac{h_{n}}{h_{n}^{2} N_{n}} \sum_{t=1}^{T_{n}} y_{n t-1}^{2}-\frac{1}{T_{n}} \sum_{t=1}^{T_{n}}\left(u_{n t}^{h}\right)^{2}\right) \stackrel{p}{\rightarrow}-\frac{2 \sigma^{2}}{3}, \\
& \frac{1}{h_{n}^{2} N_{n}} \sum_{t=1}^{T_{n}} u_{n t} u_{n t-1}=\frac{1}{T_{n}} \sum_{t=1}^{T_{n}} u_{n t}^{h} u_{n t-1}^{h} \stackrel{p}{\rightarrow} \frac{\sigma^{2}}{6} .
\end{aligned}
$$

Recall that $\sum_{t=1}^{T_{n}} y_{n t-2} y_{n t-1}=e^{\gamma h_{n}} \sum_{t=1}^{T_{n}} y_{n t-2}^{2}+\sum_{t=1}^{T_{n}} y_{n t-2} u_{n t-1}$, and it is known that

$$
h_{n} \sum_{t=1}^{T_{n}} y_{n t-2}^{2} \stackrel{p}{\rightarrow}-\frac{y(0)^{2}}{2 \gamma}, \quad \frac{1}{h_{n}^{2} N_{n}} \sum_{t=1}^{T_{n}} y_{n t-2} u_{n t-1} \stackrel{p}{\rightarrow}-\frac{2 \sigma^{2}}{3} .
$$

Note further that

$$
\begin{aligned}
& \frac{1}{h_{n}^{2} N_{n}} \sum_{t=1}^{T_{n}} y_{n t-2}^{2}=\frac{1}{h_{n}^{3} N_{n}}\left(h_{n} \sum_{t=1}^{T_{n}} y_{n t-2}^{2}\right), \\
& h_{n} \sum_{t=1}^{T_{n}} y_{n t-2} u_{n t-1}=h_{n}^{3} N_{n}\left(\frac{1}{h_{n}^{2} N_{n}} \sum_{t=1}^{T_{n}} y_{n t-2} u_{n t-1}\right) .
\end{aligned}
$$

If $h_{n}^{3} N_{n} \downarrow 0$ or $h_{n}^{3} N_{n} \rightarrow k^{\prime}$ (a constant), the appropriate normalisation is $h_{n}$, while if $h_{n}^{3} N_{n} \uparrow \infty$, the normalisation is $1 / h_{n}^{2} N_{n}$, giving the results in the lemma.

Case 3: From (A7) it follows that

$$
\begin{aligned}
\sum_{t=1}^{T_{n}} y_{n t}^{2} & =\sum_{t=1}^{T_{n}}\left(\sum_{j=1}^{t} e^{(t-j) \gamma h} u_{n j}+e^{t \gamma h} y_{n 0}\right)^{2} \\
& =\sum_{t=1}^{T_{n}}\left(\sum_{j=1}^{t} e^{(t-j) \gamma h} u_{n j}\right)^{2}+2 y(0) \sum_{t=1}^{T_{n}} e^{t \gamma h} \sum_{j=1}^{t} e^{(t-j) \gamma h} u_{n j}+y(0)^{2} \sum_{t=1}^{T_{n}} e^{2 t \gamma h} .
\end{aligned}
$$


Considering the first term,

$\frac{1}{T_{n}} \sum_{t=1}^{T_{n}}\left(\sum_{j=1}^{t} e^{(t-j) \gamma h} u_{n j}\right)^{2}=\frac{1}{T_{n}} \sum_{t=1}^{T_{n}} \sum_{j=1}^{t} e^{2(t-j) \gamma h} u_{n j}^{2}+\frac{2 e^{\gamma h}}{T_{n}} \sum_{t=1}^{T_{n}} \sum_{j=2}^{t} e^{2(t-j) \gamma h} u_{n j} u_{n j-1}+o_{p}(1)$

the $o_{p}(1)$ term corresponding to sums of terms of the form $u_{n j} u_{n j-i}$ for $i>1$, which are uncorrelated. Now, as $n \uparrow \infty$,

$$
\begin{aligned}
& \frac{1}{T_{n}} \sum_{t=1}^{T_{n}} \sum_{j=1}^{t} e^{2(t-j) \gamma h} u_{n j}^{2}=\frac{1}{T_{n}} \sum_{t=1}^{T_{n}} u_{n t}^{2} \sum_{j=0}^{T_{n}-t} e^{2 j \gamma h} \stackrel{p}{\rightarrow} \frac{\gamma_{0}(h)}{1-e^{2 \gamma h}}, \\
& \frac{1}{T_{n}} \sum_{t=1}^{T_{n}} \sum_{j=2}^{t} e^{2(t-j) \gamma h} u_{n j} u_{n j-1}=\frac{1}{T_{n}} \sum_{t=2}^{T_{n}} u_{n t} u_{n t-1} \sum_{j=0}^{T_{n}-t} e^{2 j \gamma h} \stackrel{p}{\rightarrow} \frac{\gamma_{1}(h)}{1-e^{2 \gamma h}},
\end{aligned}
$$

and hence

$$
\frac{1}{T_{n}} \sum_{t=1}^{T_{n}}\left(\sum_{j=1}^{t} e^{(t-j) \gamma h} u_{n j}\right)^{2} \stackrel{p}{\rightarrow} \frac{\gamma_{0}(h)+2 e^{\gamma h} \gamma_{1}(h)}{1-e^{2 \gamma h}} .
$$

Turning to the second term,

$$
\begin{aligned}
\frac{1}{T_{n}} y(0) \sum_{t=1}^{T_{n}} e^{t \gamma h} \sum_{j=1}^{t} e^{(t-j) \gamma h} u_{n j} & =\frac{1}{T_{n}} y(0) \sum_{t=1}^{T_{n}} e^{2 t \gamma h} \sum_{j=1}^{t} e^{-j \gamma h} u_{n j} \\
& =\frac{1}{T_{n}} y(0) \sum_{t=1}^{T_{n}} e^{-t \gamma h} u_{n t} \sum_{j=t}^{T_{n}} e^{2 j \gamma h} \\
& =\frac{1}{T_{n}} y(0) \sum_{t=1}^{T_{n}} e^{-t \gamma h} u_{n t}\left[\frac{e^{2 \gamma h}}{1-e^{2 \gamma h}}\left(e^{2(t-1) \gamma h}-e^{2 T_{n} \gamma h}\right)\right] \\
& =\frac{1}{T_{n}} y(0) \frac{e^{2 T_{n} \gamma h}}{1-e^{2 \gamma h}} \sum_{t=1}^{T_{n}} u_{n t} \stackrel{p}{\rightarrow} 0 .
\end{aligned}
$$

The third and final term is clearly $o\left(T_{n}\right)$. Combining these results yields the expression in the lemma. Next,

$$
\begin{aligned}
\frac{1}{T_{n}} \sum_{t=1}^{T_{n}} y_{n t-1} u_{n t} & =\frac{1}{T_{n}} \sum_{t=1}^{T_{n}}\left(\sum_{j=1}^{t-1} e^{(t-1-j) \gamma h} u_{n j}+e^{(t-1) \gamma h} y_{n 0}\right) u_{n t} \\
& =\frac{1}{T_{n}} \sum_{t=2}^{T_{n}} \sum_{j=1}^{t-1} e^{(t-1-j) \gamma h} u_{n j} u_{n t}+\frac{1}{T_{n}} y(0) \sum_{t=1}^{T_{n}} e^{(t-1) \gamma h} u_{n t} \\
& =\frac{1}{T_{n}} \sum_{t=2}^{T_{n}} u_{n t} u_{n t-1}+o_{p}(1) \stackrel{p}{\rightarrow} \gamma_{1}(h),
\end{aligned}
$$

since the only terms in the first summation that are uncorrelated are those for which $j=t-1$ (note that $j \leq t-1$ ). Finally, the limits for the terms determining the IV estimator follow 
from (14) and (15), noting that

$$
\frac{1}{T_{n}} \sum_{t=1}^{T_{n}} u_{n t} u_{n t-1} \stackrel{p}{\rightarrow} \gamma_{1}(h)
$$

and that $e^{\gamma h_{n}}=e^{\gamma h}=\alpha$.

Cases 3(a), 3(b): When $\gamma>0$ the result for $y(0)=0$ (case 3(a)) can be obtained as a special case of $y(0) \neq 0$ (case $3(\mathrm{~b})$ ) and hence the proof of the latter is given. The proof follows Anderson (1959), suitably adapted to allow for the moving average disturbance (Anderson deals with the purely autoregressive model). Let $\beta=\alpha^{-1}=e^{-\gamma h}$, so that $0<\beta<1$, and consider the random process

$$
z_{n t}=\beta^{t-2} y_{n t-1}=\sum_{j=1}^{t-1} \beta^{j-1} u_{n j}+\alpha y_{n 0}
$$

From equation (2.6) of Anderson (1959),

$$
\begin{aligned}
\beta^{2\left(T_{n}-2\right)} \sum_{t=1}^{T_{n}} y_{n t-1}^{2}-\left(1-\beta^{2}\right)^{-1} z_{T_{n}}^{2}= & \left(\beta^{2\left(T_{n}-2\right)} \sum_{t=1}^{T_{n}} y_{n t-1}^{2}-\frac{1-\beta^{2 T_{n}}}{1-\beta^{2}} z_{T_{n}}^{2}\right) \\
& +\left(\frac{1-\beta^{2 T_{n}}}{1-\beta^{2}} z_{T_{n}}^{2}-\frac{1}{1-\beta^{2}} z_{T_{n}}^{2}\right)
\end{aligned}
$$

and the objective is to show that this term converges to zero in probability. Since $z_{n t}=$ $z_{n t-s}+\sum_{j=1}^{s} \beta^{t-j-1} u_{n t-j}$ it follows that $z_{n T_{n}-s}-z_{n T_{n}}=-\sum_{j=1}^{s} \beta^{T_{n}-j-1} u_{n T_{n}-j}$, and it can be shown that

$$
\begin{aligned}
& E\left(z_{n T_{n}-s}-z_{n T_{n}}\right)^{2}=E\left(\sum_{j=1}^{s} \beta^{T_{n}-j-1} u_{n T_{n}-j}\right)^{2} \\
& =\left[\gamma_{0}(h)+2 \beta^{-1} \gamma_{1}(h)\right] \beta^{2\left(T_{n}-s-1\right)} \sum_{j=0}^{s-1} \beta^{2 j} \\
& \leq\left[\gamma_{0}(h)+2 \beta^{-1} \gamma_{1}(h)\right] \frac{\beta^{2\left(T_{n}-s-1\right)}}{1-\beta^{2}}, \\
& E\left(z_{n T_{n}-s}+z_{n T_{n}}\right)^{2} \leq 2 E\left(z_{n T_{n}-s}\right)^{2}+2 E\left(z_{n T_{n}}\right)^{2} \\
& \leq 4 E\left(z_{n T_{n}}\right)^{2} \\
& =4\left(\gamma_{0}(h) \sum_{j=0}^{T_{n}-2} \beta^{2 j}+2 \beta \gamma_{1}(h) \sum_{j=0}^{T_{n}-3} \beta^{2 j}+\alpha^{2} y(0)^{2}\right) \\
& \leq 4\left(\frac{\gamma_{0}(h)+2 \beta \gamma_{1}(h)}{1-\beta^{2}}+\alpha^{2} y(0)^{2}\right) \text {. }
\end{aligned}
$$

Now 


$$
\begin{aligned}
E\left|\beta^{2\left(T_{n}-2\right)} \sum_{t=1}^{T_{n}} y_{n t-1}^{2}-\frac{1-\beta^{2 T_{n}}}{1-\beta^{2}} z_{n T_{n}}^{2}\right| \leq \sum_{s=1}^{T_{n}-1} \beta^{2 s} E\left|z_{n T_{n}-s}^{2}-z_{n T_{n}}^{2}\right| \\
\quad=\sum_{s=1}^{T_{n}-1} \beta^{2 s} E\left|\left(z_{n T_{n}-s}-z_{n T_{n}}\right)\left(z_{n T_{n}-s}+z_{n T_{n}}\right)\right| \\
\quad \leq \sum_{s=1}^{T_{n}-1} \beta^{2 s}\left[E\left(z_{n T_{n}-s}-z_{n T_{n}}\right)^{2} E\left(z_{n T_{n}-s}+z_{n T_{n}}\right)^{2}\right]^{1 / 2} \\
\quad \leq 2\left(\frac{\gamma_{0}(h)+2 \beta \gamma_{1}(h)}{1-\beta^{2}}+\alpha^{2} y(0)^{2}\right)^{1 / 2}\left(\frac{\gamma_{0}(h)+2 \beta^{-1} \gamma_{1}(h)}{1-\beta^{2}}\right)^{1 / 2} \sum_{s=1}^{T_{n}-1} \beta^{T_{n}+s-1} \\
\quad \leq 2\left(\frac{\gamma_{0}(h)+2 \beta \gamma_{1}(h)}{1-\beta^{2}}+\alpha^{2} y(0)^{2}\right)^{1 / 2}\left(\frac{\gamma_{0}(h)+2 \beta^{-1} \gamma_{1}(h)}{1-\beta^{2}}\right)^{1 / 2} \frac{\beta^{T_{n}}}{1-\beta},
\end{aligned}
$$

so that, by Chebyshev's inequality,

$$
\operatorname{Pr}\left(\left|\beta^{2\left(T_{n}-2\right)} \sum_{t=1}^{T_{n}} y_{n t-1}^{2}-\frac{1-\beta^{2 T_{n}}}{1-\beta^{2}} z_{n T_{n}}^{2}\right|>\epsilon\right) \leq \frac{K}{\epsilon} \beta^{T_{n}} \rightarrow 0
$$

as $n \uparrow \infty$, while

$$
E\left(\frac{\beta^{2 T_{n}}}{1-\beta^{2}} z_{n T_{n}}^{2}\right) \leq C \beta^{2 T_{n}} \rightarrow 0
$$

as $n \uparrow \infty$, thus establishing that

$$
\beta^{2\left(T_{n}-2\right)} \sum_{t=1}^{T_{n}} y_{n t-1}^{2}-\left(1-\beta^{2}\right)^{-1} z_{n T_{n}}^{2} \stackrel{p}{\rightarrow} 0 .
$$

Now $z_{n T_{n}} \sim \mathrm{N}\left(\alpha y(0), \sigma_{z n}^{2}\right)$ where

$$
\sigma_{z n}^{2}=\gamma_{0}(h) \sum_{j=0}^{T_{n}-2} \beta^{2 j}+2 \beta \gamma_{1}(h) \sum_{j=0}^{T_{n}-3} \beta^{2 j}
$$

is derived from the earlier definition of $z_{n t}$. As $n \uparrow \infty, \sigma_{z n}^{2} \rightarrow \theta_{h}^{2}$ and the limiting distribution of $z_{n T_{n}}$ has the representation $\alpha y(0)+\theta_{h} \eta$.

Turning to the numerator of $\widehat{\alpha}_{n}-\alpha$, it is convenient to introduce the random process $x_{n t}=\sum_{j=0}^{t-1} \beta^{j} u_{n t-j}$ and to consider the difference

$$
\beta^{T_{n}-2} \sum_{t=1}^{T_{n}} y_{n t-1} u_{n t}-x_{n T_{n}} z_{n T_{n}}=\sum_{s=1}^{T_{n}-1} \beta^{s} u_{n T_{n}-s}\left(z_{n T_{n}-s}-z_{n T_{n}}\right) .
$$

Then, using earlier results, 


$$
\begin{aligned}
E & \left|\beta^{T_{n}-2} \sum_{t=1}^{T_{n}} y_{n t-1} u_{n t}-x_{n T_{n}} z_{n T_{n}}\right| \leq \sum_{s=1}^{T_{n}-1} \beta^{s} E\left|u_{n T_{n}-s}\left(z_{n T_{n}-s}-z_{n T_{n}}\right)\right| \\
& \leq \sum_{s=1}^{T_{n}-1} \beta^{s}\left(E u_{n T_{n}-s}^{2} E\left(z_{n T_{n}-s}-z_{n T_{n}}\right)^{2}\right)^{1 / 2} \\
& \leq \sum_{s=1}^{T_{n}-1} \beta^{s}\left(\gamma_{0}(h)\left[\gamma_{0}(h)+2 \beta^{-1} \gamma_{1}(h)\right] \frac{\beta^{2\left(T_{n}-s-1\right)}}{1-\beta^{2}}\right)^{1 / 2} \\
& \leq\left(\frac{\gamma_{0}(h)\left[\gamma_{0}(h)+2 \beta^{-1} \gamma_{1}(h)\right]}{1-\beta^{2}}\right)^{1 / 2} \sum_{s=1}^{T_{n}-1} \beta^{T_{n}-1} \\
& =\left(\frac{\gamma_{0}(h)\left[\gamma_{0}(h)+2 \beta^{-1} \gamma_{1}(h)\right]}{1-\beta^{2}}\right)^{1 / 2}\left(T_{n}-1\right) \beta^{T_{n}-1},
\end{aligned}
$$

so that by Chebyshev's inequality,

$$
\operatorname{Pr}\left(\left|\beta^{T_{n}-2} \sum_{t=1}^{T_{n}} y_{n t-1} u_{n t}-x_{n T_{n}} z_{n T_{n}}\right|>\epsilon\right) \leq \frac{K}{\epsilon}\left(T_{n}-1\right) \beta^{T_{n}-1} \rightarrow 0
$$

as $n \uparrow \infty$, and so $\beta^{T_{n}-2} \sum_{t=1}^{T_{n}} y_{n t-1} u_{n t}-x_{n T_{n}} z_{n T_{n}} \stackrel{p}{\rightarrow} 0$. Now, from the definition of $x_{n t}$ it follows that $x_{n T_{n}} \sim \mathrm{N}\left(0, \sigma_{x n}^{2}\right)$, where

$$
\sigma_{x n}^{2}=\gamma_{0}(h) \sum_{j=0}^{T_{n}-1} \beta^{2 j}+2 \beta \gamma_{1}(h) \sum_{j=0}^{T_{n}-2} \beta^{2 j}
$$

As $n \uparrow \infty, \sigma_{x n}^{2} \rightarrow \theta_{h}^{2}$ and the limiting distribution of $x_{n T_{n}}$ has the representation $\theta_{h} \xi$. Furthermore, the two variables $x_{n T_{n}}$ and $z_{n T_{n}}$ are uncorrelated in the limit since

$$
E\left(x_{n T_{n}} z_{n T_{n}}\right)=\beta^{T_{n}-1}\left(T_{n}-1\right) \gamma_{0}(h)+\beta^{T_{n}-2}\left(T_{n}-1\right) \gamma_{1}(h)+\beta^{T_{n}}\left(T_{n}-2\right) \gamma_{0}(h) \rightarrow 0
$$

as $n \uparrow \infty$. Finally, for the IV estimator, the stated limit for $\sum_{t=1}^{T_{n}} y_{n t-2} y_{n t-1}$ follows from (15) since

$$
\frac{\left(\alpha^{2}-1\right)}{\alpha^{2\left(T_{n}-1\right)}} \sum_{t=1}^{T_{n}} y_{n t-2} u_{n t-1} \stackrel{p}{\rightarrow} 0,
$$

while the limit for $\sum_{t=1}^{T_{n}} y_{n t-2} u_{n t}$ follows because

$$
\frac{1}{\alpha^{\left(T_{n}-2\right)}} \sum_{t=1}^{T_{n}} u_{n t} u_{n t-1} \stackrel{p}{\rightarrow} 0
$$

This completes the proof. 
Proof of Theorem 3. The same approach as in the proof of Theorem 2 is followed, based here on the limiting behaviour of the sample moments established in Lemma 2 .

Case 1(a): From Lemma 2,

$$
\frac{1}{h_{n}}\left(\widehat{\alpha}_{n}-\alpha_{n}\right) \Rightarrow \frac{\Omega(J, W, 1 / 6)}{N \Psi\left(J^{2}\right)}, \frac{1}{h_{n}}\left(\widetilde{\alpha}_{n}-\alpha_{n}\right) \Rightarrow \frac{\Omega(J, W, 0)}{N \Psi\left(J^{2}\right)} .
$$

Multiplying by $N$ yields the distributions of $T_{n}\left(\widehat{\alpha}_{n}-\alpha_{n}\right)$ and $T_{n}\left(\widetilde{\alpha}_{n}-\alpha_{n}\right)$. Now $T_{n}\left(\widehat{\alpha}_{n}-1\right)=$ $T_{n}\left(\widehat{\alpha}_{n}-\alpha_{n}\right)+T_{n}\left(\alpha_{n}-1\right)$ and $T_{n}\left(\widetilde{\alpha}_{n}-1\right)=T_{n}\left(\widetilde{\alpha}_{n}-\alpha_{n}\right)+T_{n}\left(\alpha_{n}-1\right)$, so it is necessary to examine the behaviour of $T_{n}\left(\alpha_{n}-1\right)$ as $n \uparrow \infty$. Since $\alpha_{n}=e^{\gamma h_{n}}=e^{\gamma N / T_{n}}$,

$$
T_{n}\left(\alpha_{n}-1\right)=T_{n}\left(e^{\gamma N / T_{n}}-1\right)=T_{n}\left(\frac{\gamma N}{T_{n}}+\frac{1}{2}\left(\frac{\gamma N}{T_{n}}\right)^{2}+O\left(T_{n}^{-3}\right)\right) \rightarrow \gamma N
$$

as $n \uparrow \infty$. This yields the stated results.

Case 1(b): By a similar argument as in case $1(\mathrm{a}), h_{n}^{-1}\left(\widehat{\alpha}_{n}-\alpha_{n}\right) \stackrel{p}{\rightarrow} 0$ and $h_{n}^{-1}\left(\widetilde{\alpha}_{n}-\alpha_{n}\right) \stackrel{p}{\rightarrow} 0$, from which the results follow using (A10).

Case 2(a): When $\gamma>0$, Lemma 2 yields the normalisation factor for $\widehat{\alpha}_{n}-\alpha_{n}$ and $\widetilde{\alpha}_{n}-$ $\alpha_{n}$ as $\left(2 \gamma N_{n} / e^{\gamma N_{n}} h_{n}^{2} N_{n}\right) /\left(\left(2 \gamma N_{n}\right)^{2} / e^{2 \gamma N_{n}} h_{n} N_{n}^{2}\right)=e^{\gamma N_{n}} T_{n} / 2 \gamma N_{n}$, while that for $\widehat{\alpha}_{n}-1$ and $\widetilde{\alpha}_{n}-1$ is $T_{n}\left(\alpha_{n}-1\right)=\gamma N_{n}$ using (A10). When $\gamma<0$, the normalisation factor is $\left(\left(-2 \gamma N_{n}\right)^{1 / 2} / h_{n}^{2} N_{n}\right) /\left(-2 \gamma N_{n} / h_{n} N_{n}^{2}\right)=T_{n} /\left(-2 \gamma N_{n}\right)^{1 / 2}$ for the OLS estimator, and $\left(1 / h_{n}^{2} N_{n}\right) /\left(-2 \gamma N_{n} / h_{n} N_{n}^{2}\right)=T_{n} /\left(-2 \gamma N_{n}\right)$ for the IV estimator. The results follow straightforwardly.

Cases 2(b), 2(b)(i), 2(b)(ii): The normalisation derived from Lemma 2 is $T_{n} / N_{n}$ in all these cases, and use is also made of the fact that $\left(T_{n} / N_{n}\right)\left(\alpha_{n}-1\right)=\gamma$ using (A10).

Case 2(b)(iii): In all cases except where $h_{n}^{3} N_{n} \uparrow \infty$, the normalisation derived from Lemma 2 is $T_{n}^{3} / N_{n}^{4}$. Also, note that

$$
\frac{T_{n}^{3}}{N_{n}^{4}}\left(\alpha_{n}-1\right)=\frac{1}{h_{n}^{2} N_{n}}\left(\frac{T_{n}}{N_{n}}\right)\left(\alpha_{n}-1\right) \downarrow 0
$$

as $n \uparrow \infty$ using (A10) and because $h_{n} N_{n}^{1 / 2} \uparrow \infty$. In the final case, the normalisation is unity and note that $\alpha_{n}-1=e^{\gamma h_{n}}-1 \downarrow 0$ as $n \uparrow \infty$ because $h_{n} \downarrow 0$.

Case 3: The stated probability limits follow straightforwardly from Lemma 2 and from noting that $\alpha-1=e^{\gamma h}-1$ is a constant.

Cases 3(a), 3(b): The scaling in these cases is $\alpha^{T_{n}-2} /\left(1-\alpha^{-2}\right)=\alpha^{T_{n}} /\left(\alpha^{2}-1\right)$, and the distributions are obtained as the limit of $x_{n T_{n}} / z_{n T_{n}}$, where $x_{n T_{n}}$ and $z_{n T_{n}}$ are defined in the proof of Lemma 2. Noting that $\alpha-1=e^{\gamma h}-1$ is a constant yields the expressions for the deviations around unity. 
Proof of Theorem 4. In cases where $T_{n}\left(\widehat{\alpha}_{n}-1\right)$ and $T_{n}\left(\widetilde{\alpha}_{n}-1\right)$ (or some other suitably normalised statistics) are $O_{p}(1)$ under the null $\gamma=0$ but converge in probability to $\pm \infty$ (depending on the sign of $\gamma$ ) under the alternative, the tests are clearly consistent. This occurs in cases 1(b), 2(a), 2(b)(i), 2(b)(ii), most of 2(b)(iii) and 3, and is easily verfied by the stated distributions under the alternative given in Theorem 3. In case 3, when $\gamma<0$, some extra work is needed to show that $T_{n}\left(\widehat{\alpha}_{n}-1\right) \stackrel{p}{\rightarrow}-\infty$, which follows if it can be shown that the probability limit of $\left(\widehat{\alpha}_{n}-\alpha\right)$ plus the term $\alpha-1$ is negative. This will now be demonstrated. From Theorem 1,

$$
\begin{aligned}
\gamma_{0}(h)+2 e^{\gamma h} \gamma_{0}(h) & =\frac{\sigma^{2}}{\gamma^{2}}\left(h\left(e^{2 \gamma h}+1\right)+\frac{\left(1-e^{2 \gamma h}\right)}{h}\right)+\frac{2 e^{\gamma h} \sigma^{2}}{\gamma^{2}}\left(\frac{e^{2 \gamma h}-1}{2 \gamma}-h e^{\gamma h}\right) \\
& =\frac{\sigma^{2}}{\gamma^{2}}\left(1-e^{2 \gamma h}\right)\left(h+\frac{1}{h}-\frac{e^{\gamma h}}{\gamma}\right),
\end{aligned}
$$

while

$$
\left(1-e^{2 \gamma h}\right) \gamma_{1}(h)=-\frac{\sigma^{2}}{\gamma^{2}} \frac{\left(1-e^{2 \gamma h}\right)}{2 \gamma}\left[\left(1-e^{2 \gamma h}\right)+2 \gamma h e^{\gamma h}\right]
$$

Defining $x=\gamma h<0$ and $c_{h}=1+h^{-2}>0$, the term of interest may be written

$$
\begin{aligned}
\frac{\left(1-e^{2 \gamma h}\right) \gamma_{1}(h)}{\gamma_{0}(h)+2 e^{\gamma h} \gamma_{1}(h)}+e^{\gamma h}-1 & =\frac{-\left[\left(1-e^{2 x}\right)+2 x e^{x}\right]-\left(1-e^{x}\right)\left(2 c_{h} x-2 e^{x}\right)}{2 c_{h} x-2 e^{x}} \\
& =\frac{f(x)}{2\left(c_{h} x-e^{x}\right)} .
\end{aligned}
$$

Clearly, the denominator is negative, while the numerator $f(x)$ may be simplified as $f(x)=$ $2 x c_{h}\left(e^{x}-1\right)-2 x e^{x}-\left(1-e^{x}\right)^{2}$. Then $f(x) \geq 0$ if

$$
c_{h}=1+h^{-2} \geq \frac{e^{x}-1}{2 x}+\frac{e^{x}}{e^{x}-1} .
$$

In fact, since $c_{h} \geq 1$, it is sufficient to show that the term on the right hand side is $\leq 1$, which in turn requires $g(x)=\left(e^{x}-1\right)^{2}+2 x \leq 0$. Note that $g(0)=0$ while $g^{\prime}(x)=2+2\left(e^{x}-1\right) e^{x}>0$ for $x<0$, implying that $g(x) \leq 0$ for $x \leq 0$ and $f(x) \geq 0$, as required. The only cases where there does not exist a consistent test are case $1(\mathrm{a})$, where the statistics are $O_{p}(1)$ under both the null and the alternative, and in case 2(b)(iii) when $h_{n}^{3} N_{n} \uparrow \infty$. In the latter case, $T_{n}\left(\widetilde{\alpha}_{n}-1\right) \rightarrow \infty$ when $\gamma<0$, and hence the test is not consistent against such an alternative. 


\section{References}

Anderson, T. W. (1959) On asymptotic distributions of estimates of parameters of stochastic difference equations. Annals of Mathematical Statistics 30, 676-687.

Bergstrom, A. R. (1984) Continuous time stochastic models and issues of aggregation over time. In Z. Griliches and M. D. Intriligator (eds.), Handbook of Econometrics, Volume 2, pp. 1145-1212. North-Holland, Amsterdam.

Choi, I. (1992) Effects of data aggregation on the power of tests for a unit root. Economics Letters 40, 397-401.

Hall, A. (1989) Testing for a unit root in the presence of moving average errors. Biometrika $76,49-56$.

Ng, S. (1995) Testing for unit roots in flow data sampled at different frequencies. Economics Letters 47, 237-242.

Perron, P. (1991) Test consistency with varying sampling frequency. Econometric Theory 7, $341-368$.

Phillips, P. C. B. (1987a) Time series regression with a unit root. Econometrica 55, 277-301.

Phillips, P. C. B. (1987b) Towards a unified asymptotic theory for autoregression. Biometrika $74,535-547$. 